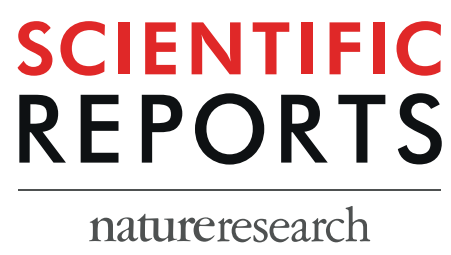

\title{
OPEN Diagnostic performance of hematological discrimination indices to discriminate between Beta thalassemia trait and iron deficiency anemia and using cluster analysis: Introducing two new indices tested in Iranian population
}

Mina Jahangiri ${ }^{1,2}$, Fakher Rahim $\mathbb{1}^{3,4^{*}}$ \& Amal Saki Malehi ${ }^{1,3}$

Although the discrimination between $\beta$-thalassemia trait ( $\beta$ TT) and Iron deficiency anemia (IDA) is important clinically, but it is challenging and normally difficult; so if a patient with IDA is diagnosed as $\beta T T$, then it is deprived of iron therapy. This study purpose was to evaluate the 26 different discriminating indices diagnostic function in patients with microcytic anemia by using accuracy measures, and also recommending two distinct new discriminating indices as well. In this study, 907 patients were enrolled with the ages over 18-year-old with either $\beta$ TT or IDA. Twenty-six discrimination indices diagnostic performance presented in earlier studies, and two new indices were introduced in this study (CRUISE index and index26) in order to evaluate the differential between $\beta$ TT and IDA by using accuracy measures. 537 (59\%) patients with $\beta T T$ ( 299 (56\%) women, and $238(44 \%)$ men), and also $370(41 \%)$ patients with IDA (293 (79\%) women, and $77(21 \%)$ men) were participated in this study for evaluating the 28 discrimination indices diagnostic performance. Two new introduced indices (CRUISE index and index26) have better performance than some discrimination indices. Indices with the amount of AUC higher than 0.8 had very appropriate diagnostic accuracy in discrimination between $\beta T T$ and IDA, and also CRUISE index has good diagnostic accuracy, too. The present study was also the first cluster analysis application in order to identify the homogeneous subgroups of different indices with similar diagnostic function. In addition, new indices that offered in this study have presented a relatively closed diagnostic performance by using cluster analysis for the different indices described in earlier studies. Thus, we suggest the using of cluster analysis in order to determine differential indices with similar diagnostic performances.

$\beta$-thalassemia trait ( $\beta$ TT) and iron deficiency anemia (IDA) are amongst the most regularly reported microcytic anemia disorders ${ }^{1,2}$. IDA is prevalent in developing countries, hence $\beta$ TT is predominant in regions like the Mediterranean, the Middle East, and the South East $^{3-7}$. However the discrimination between $\beta$ TT and IDA is important clinically, but it is challenging and normally difficult, because both of the disorders are sometimes clinically and experimentally in the similar conditions ${ }^{8-10}$. Thus, if a patient with IDA is identified as $\beta$ TT, then he is deprived of iron therapy. Considering that $\beta$ TT does not need treatment, but the diagnosis of a patient with $\beta$ TT,

${ }^{1}$ Department of Biostatistics and Epidemiology, Faculty of Health, Ahvaz Jundishapur University of Medical Sciences, Ahvaz, Iran. ${ }^{2}$ Ph.D. Student, Department of Biostatistics, Faculty of Medical Sciences, Tarbiat Modares University, Tehran, Iran. ${ }^{3}$ Research Center of Thalassemia and Hemoglobinopathy, Health Research Institute, Ahvaz Jundishapur University of Medical Sciences, Ahvaz, Iran. ${ }^{4}$ Clinical Research Development Unit, Golestan Hospital, Ahvaz Jundishapur University of Medical Sciences, Ahvaz, Iran. *email: Bioinfo2003@gmail.com 


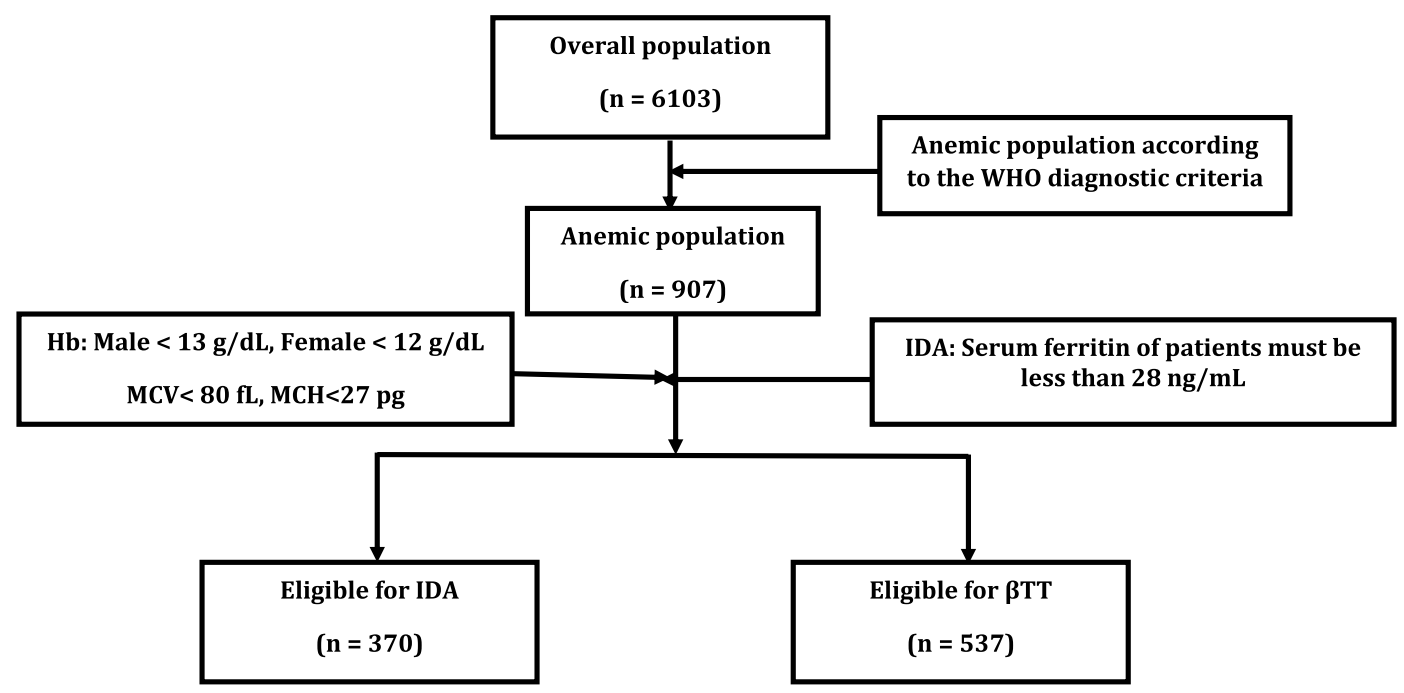

Figure 1. Design of study used for the validation of the CRUISE index and index26. Hb: hemoglobin; MCV: mean corpuscular volume; MCH: mean corpuscular hemoglobin; IDA: iron deficiency anemia; $\beta$ TT: $\beta$ eta thalassemia trait.

\begin{tabular}{|c|c|c|c|c|c|}
\hline & \multicolumn{2}{|l|}{$\beta T T(n=537)$} & \multicolumn{2}{|l|}{ IDA $(n=370)$} & \multirow[b]{2}{*}{$P$-value } \\
\hline & Mean \pm SD & Median (IQR) & Mean \pm SD & Median (IQR) & \\
\hline Age & $21.98 \pm 16.37$ & $20(24)$ & $28.86 \pm 14.58$ & $27(22.75)$ & $<0.001$ \\
\hline $\mathrm{MCV}$ & $62.17 \pm 4.14$ & $62(5.4)$ & $71.87 \pm 6.93$ & $72.2(9.73)$ & $<0.001$ \\
\hline $\mathrm{MCH}$ & 19.751 .45 & $.196(1.8)$ & $21.85 \pm 2.99$ & $21.9(4.2)$ & $<0.001$ \\
\hline $\mathrm{MCHC}$ & $31.71 \pm 1.48$ & $31.84(1.43)$ & $30.40 \pm 3.04$ & $30.3(2.71)$ & $<0.001$ \\
\hline $\mathrm{Hb}$ & $11.20 \pm 1.41$ & $11(1.16)$ & $10.82 \pm 2.43$ & $10.45(2.62)$ & $<0.001$ \\
\hline $\mathrm{HCT}$ & $35.39 \pm 4.73$ & $34.6(5.15)$ & $35.53 \pm 6.71$ & $34(7.65)$ & 0.182 \\
\hline RDW & $15.88 \pm 1.43$ & $15.7(1.7)$ & $16.04 \pm 2.31$ & $15.7(3.32)$ & 0.94 \\
\hline $\mathrm{RBC}$ & $5.69 \pm 0.67$ & $5.61(0.93)$ & $4.91 \pm 0.69$ & $4.83(0.83)$ & $<0.001$ \\
\hline $\mathrm{HbA} 2$ & $5.09 \pm 0.74$ & $5(1.1)$ & $2.43 \pm 0.63$ & $2.4(0.83)$ & $<0.001$ \\
\hline Serum Iron & $85.05 \pm 32.96$ & $86(47)$ & $25.66 \pm 8.21$ & $25(13)$ & $<0.001$ \\
\hline TIBC & $346.35 \pm 47.02$ & $345(54)$ & $480 \pm 25.77$ & $466(40)$ & $<0.001$ \\
\hline Serum Ferritin & $55.44 \pm 56.64$ & $38.9(53.9)$ & $4.52 \pm 1.85$ & $4.3(2.3)$ & $<0.001$ \\
\hline
\end{tabular}

Table 1. Descriptive statistics of hematological parameters and age variable of study groups (IDA and $\beta$ TT).

and IDA may cause attendant risk of birth of thalassemia major child in the pre-marriage genetic counseling ${ }^{11-13}$. To effectively differentiate between these two hematologic disorders, in addition to counting blood cells (CBC), also time-consuming, and cost-effective tests are essential. Because the definitive diagnosis between $\beta$ TT and IDA is confirmed by performing blood tests in order to measure the HbA2, serum iron, serum ferritin, transferrin saturation, and total iron binding capacity (TIBC), and in fact these parameters are typically considered as the gold standards for discriminating between these two hematologic disorders ${ }^{9,14-18}$.

Because of the discriminating between these two disorders importance, and cost-effective and time-consuming tests in order to differentiate them, several discriminating indicators have been proposed in large-scale research for the rapid and inexpensive differentiation between these two common hematologic disorders since 1973. These indices are founded on the blood parameters obtained from automated cell counters of blood that traditionally derived parameters of $\mathrm{Hb}$ (Hemoglobin), Mean Corpuscular Volume (MCV), Mean Corpuscular Hemoglobin $(\mathrm{MCH})$, Red Blood Cell Distribution Width (RDW), Mean Corpuscular Hemoglobin Concentration (MCHC), and Red Blood Cell Count (RBC) ${ }^{19-41}$. Several studies have studied these indices diagnostic accuracy, which presented different results, as well as none of these indicators showed a sensitivity and specificity of $100 \% 0^{3,6,17,32,40,42-56}$. Therefore, this study purpose was to evaluate the diagnostic function of 26 different discriminating indices in patients with microcytic anemia, by using accuracy measures, and proposing two distinct new discriminating indices for differentiation between $\beta$ TT and IDA, as well. 


\begin{tabular}{|c|c|c|c|c|}
\hline Discriminant Formula & Reference & Calculation & $\begin{array}{l}\text { Cut-off } \\
\text { BTT }\end{array}$ & $\begin{array}{l}\text { Cut-off } \\
\text { IDA }\end{array}$ \\
\hline England and Fraser (E\&F) & 19 & $\mathrm{MCV}-\mathrm{RBC}-(5 \mathrm{HB})-3.4$ & $<0$ & $>0$ \\
\hline $\mathrm{RBC}$ & 20 & $\mathrm{RBC}$ & $>5$ & $<5$ \\
\hline Mentzer & 21 & MCV/RBC & $<13$ & $>13$ \\
\hline Srivastava & 22 & $\mathrm{MCH} / \mathrm{RBC}$ & $<3.8$ & $>3.8$ \\
\hline Shine and Lal (S\&L) & 23 & $\mathrm{MCV} \times \mathrm{MCH} \times 0 / 01$ & $<1530$ & $>1530$ \\
\hline Bessman & 24 & RDW & $<14$ & $>14$ \\
\hline Ricerca & 25 & RDW/RBC & $<4.4$ & $>4.4$ \\
\hline Green and King (G\&K) & 26 & $\left(\mathrm{MCV}^{2} \times \mathrm{RDW}\right) /(100 \mathrm{HB})$ & $<65$ & $>65$ \\
\hline Das Gupta & 27 & $1.89 \mathrm{RBC}-0.33 \mathrm{RDW}-3.28$ & $>0$ & $<0$ \\
\hline Jayabose (RDWI) & 28 & $(\mathrm{MCV} \times \mathrm{RDW}) / \mathrm{RBC}$ & $<220$ & $>220$ \\
\hline Telmissani - MCHD & 29 & $\mathrm{MCH} / \mathrm{MCV}$ & $<0.34$ & $>0.34$ \\
\hline Telmissani - MDHL & 29 & $(\mathrm{MCH} \times \mathrm{RBC}) / \mathrm{MCV}$ & $>1.75$ & $<1.75$ \\
\hline Huber- Herklotz & 30 & $(\mathrm{MCH} \times \mathrm{RDW} / 10 \mathrm{RBC})+\mathrm{RDW}$ & $<20$ & $>20$ \\
\hline Kerman I & 31 & $(\mathrm{MCV} \times \mathrm{MCH}) / \mathrm{RBC}$ & $<300$ & $300-400$ \\
\hline Kerman II & 31 & $(\mathrm{MCV} \times \mathrm{MCH} \times 10) /(\mathrm{RBC} \times \mathrm{MCHC})$ & $<85$ & $85-105$ \\
\hline Sirdah & 32 & $\mathrm{MCV}-\mathrm{RBC}-(3 \mathrm{Hb})$ & $<27$ & $>27$ \\
\hline Ehsani & 33 & $\mathrm{MCV}-(10 \mathrm{RBC})$ & $<15$ & $>15$ \\
\hline Keikhaei & 34 & $(\mathrm{HB} \times \mathrm{RDW} \times 100) /\left(\mathrm{RBC}^{2} \times \mathrm{MCHC}\right)$ & $<21$ & $>21$ \\
\hline Nishad & 35 & $0.615 \mathrm{MCV}+0.518 \mathrm{MCH}+0.446 \mathrm{RDW}$ & $<59$ & $>59$ \\
\hline Wongprachum & 36 & $(\mathrm{MCV} \times \mathrm{RDW} / \mathrm{RBC})-10 \mathrm{HB}$ & $<104$ & $>104$ \\
\hline Sehgal & 37 & $\mathrm{MCV}^{2} / \mathrm{RBC}$ & $<972$ & $>972$ \\
\hline Pornprasert & 38 & MCHC & $<31$ & $>31$ \\
\hline Sirachainan & 39 & $1.5 \mathrm{HB}-0.05 \mathrm{MCV}$ & $>14$ & $<14$ \\
\hline Bordbar & 40 & $|80-\mathrm{MCV}| \times|27-\mathrm{MCH}|$ & $>44.76$ & $<44.76$ \\
\hline Matos and Carvalho (MC) & 64 & $1.91 \mathrm{RBC}+0.44 \mathrm{MCHC}$ & $>23.85$ & $<23.85$ \\
\hline Janel $(11 \mathrm{~T})$ & 41 & $\begin{array}{l}\text { Combination of RBC, Mentzer, S\&L, E\&F, Srivastava, } \\
\text { G\&K, RDW, RDWI, Ricerca, Ehsani and Sirdah }\end{array}$ & $\geq 8$ & $<8$ \\
\hline CRUISE & \multicolumn{2}{|c|}{$\mathrm{MCHC}+0.603 \mathrm{RBC}+0.523 \mathrm{RDW}$} & $\geq 42.63$ & $<42.63$ \\
\hline Index 26 & \multicolumn{2}{|c|}{ Combination of all indices except Janel $(11 \mathrm{~T})$ index } & $\geq 16$ & $<16$ \\
\hline
\end{tabular}

Table 2. Discrimination indices for differential between $\beta T T(n=537)$ and IDA $(n=370)$ in patients with microcytic anemia.

\section{Material and Methods}

Population evaluated to develop the new index. In this study, a total of 907 patients aged over 18 years old diagnosed with IDA or $\beta$ TT were selected to develop new discriminating indices. Hematological parameters like Hb (Hemoglobin), Mean Corpuscular Volume (MCV), Mean Corpuscular Hemoglobin (MCH), Red Blood Cell Distribution Width (RDW), Mean Corpuscular Hemoglobin Concentration (MCHC), and Red Blood Cell count (RBC) were measured by using Sysmex kx-21 automated hematology analyzer.

Inclusion criteria. In the IDA group, patients had hemoglobin (Hb) levels less than 12 and $13 \mathrm{~g} / \mathrm{dL}$ for women and men, respectively. Mean corpuscular hemoglobin (MCH) and Mean corpuscular volume (MCV) were below $80 \mathrm{fL}$ and $27 \mathrm{pg}$ for both sexes, respectively, and for men, ferritin of $<28 \mathrm{ng} / \mathrm{mL}$ was considered as IDA. In the $\beta$ TT group, patients had a MCV value below $80 \mathrm{fL}$. Patients with $\mathrm{HbA} 2$ levels of $>3.5 \%$ were considered as $\beta$ TT carriers.

Exclusion criteria. For the IDA group, patients who had mutations associated with $\alpha \mathrm{TT}$ (3.7, 4.2, 20.5, MED, SEA, THAI, FIL, and Hph) were excluded so, individuals presenting the two diseases simultaneously were not selected. For the $\beta$ TT group, patients with $\alpha$ TT confirmed by presence of mutations in molecular analysis were excluded. All patients with malignancies or inflammatory/infectious diseases diagnosed based on clinical data and personal information obtained from medical records were also excluded.

Ethical consideration. This study was approved and supported by Ethical committee affiliated by the Ahvaz Jundishapur University of Medical Sciences (AJUMS), Ahvaz, Iran. A written informed consent was obtained before the enrollment. All methods were performed in accordance with the relevant guidelines and the institution regulations.

Development of the new index. 26 discrimination indices of diagnostic performance proposed in the literature, and 2 new indices introduced in this study (CRUISE index and index26) were considered for evaluation of differences between $\beta$ TT and IDA using accuracy measures like sensitivity, specificity, false positive and 


\begin{tabular}{|c|c|c|c|c|c|c|}
\hline \multicolumn{2}{|l|}{ Discriminant Formula } & \multirow{2}{*}{\begin{tabular}{|l|} 
TP \\
338 \\
\end{tabular}} & \multirow{2}{*}{\begin{tabular}{|l|} 
FP \\
54 \\
\end{tabular}} & \multirow{2}{*}{\begin{tabular}{|l|} 
FN \\
199 \\
\end{tabular}} & \multirow{2}{*}{\begin{tabular}{|l|} 
TN \\
316 \\
\end{tabular}} & \multirow{3}{*}{\begin{tabular}{|l}
$(\mathrm{TP}+\mathrm{TN})$ \\
654
\end{tabular}} \\
\hline \multirow{2}{*}{ England and Fraser (E\&F) } & $\beta \mathrm{TM}$ & & & & & \\
\hline & IDA & 316 & 199 & 54 & 338 & \\
\hline \multirow{2}{*}{$\mathrm{RBC}$} & $\beta \mathrm{TM}$ & 464 & 137 & 73 & 223 & \multirow{2}{*}{687} \\
\hline & IDA & 223 & 73 & 137 & 464 & \\
\hline \multirow{2}{*}{ Mentzer } & $\beta$ TM & 478 & 79 & 59 & 291 & \multirow{2}{*}{769} \\
\hline & IDA & 291 & 59 & 79 & 478 & \\
\hline \multirow{2}{*}{ Srivastava } & $\beta T M$ & 402 & 71 & 135 & 299 & \multirow{2}{*}{701} \\
\hline & IDA & 299 & 135 & 71 & 402 & \\
\hline \multirow{2}{*}{ Shine and Lal (S\&L) } & $\beta \mathrm{TM}$ & 537 & 305 & 0 & 65 & \multirow{2}{*}{842} \\
\hline & IDA & 65 & 0 & 305 & 537 & \\
\hline \multirow{2}{*}{ Bessman } & $\beta \mathrm{TM}$ & 34 & 74 & 503 & 296 & \multirow{2}{*}{330} \\
\hline & IDA & 296 & 503 & 74 & 34 & \\
\hline Bicerca & $\beta$ TM & 530 & 344 & 7 & 26 & 556 \\
\hline Kicerca & IDA & 26 & 7 & 344 & 530 & 536 \\
\hline Green and King (G\&K) & $\beta$ TM & 465 & 79 & 72 & 291 & 756 \\
\hline Green and King (G\&K) & IDA & 291 & 72 & 79 & 465 & 156 \\
\hline Das Gunta & $\beta$ TM & 512 & 236 & 25 & 134 & 646 \\
\hline Das Gupta & IDA & 134 & 25 & 236 & 512 & 646 \\
\hline Javabose (RDWI) & $\beta \mathrm{TM}$ & 497 & 132 & 40 & 238 & 735 \\
\hline Jayabose (RDWI) & IDA & 238 & 40 & 132 & 497 & 735 \\
\hline Telmissani-MCHD & $\beta$ TTM & 528 & 357 & 9 & 13 & 541 \\
\hline Telmissanı-MCHD & \begin{tabular}{|l|} 
IDA \\
\end{tabular} & 13 & 9 & 357 & 528 & 541 \\
\hline Telmiscani-MDHI & 及TM & 303 & 53 & 234 & 317 & 620 \\
\hline Ielmissani - MIDHL & IDA & 317 & 234 & 53 & 303 & 620 \\
\hline Huher-Herklotr & $\beta$ TTM & 121 & 52 & 416 & 318 & 439 \\
\hline Huber - HerklotZ & IDA & 318 & 416 & 52 & 121 & 439 \\
\hline & $\beta$ TTM & 507 & \begin{tabular}{|l|l}
141 \\
\end{tabular} & 30 & 229 & \\
\hline Kerman 1 & IDA & 229 & 30 & 141 & 507 & 736 \\
\hline & $\beta$ TTM & 476 & 66 & 61 & 304 & \\
\hline Kerman II & IDA & 304 & 61 & 66 & 476 & 780 \\
\hline & $\beta \mathrm{TMM}$ & 431 & 42 & 106 & 328 & \\
\hline Sirdah & \begin{tabular}{|l} 
IDA \\
\end{tabular} & 328 & 106 & 42 & 431 & 759 \\
\hline & $\beta$ TTM & 478 & 69 & 59 & 301 & \\
\hline Ehsani & \begin{tabular}{|l} 
IDA \\
\end{tabular} & 301 & 59 & \begin{tabular}{|l|}
69 \\
\end{tabular} & 478 & 779 \\
\hline & $\beta$ TM & 476 & 101 & 61 & 269 & \\
\hline Keikhaei & \begin{tabular}{|l} 
IDA \\
\end{tabular} & 269 & 61 & 101 & 476 & 745 \\
\hline & $\beta$ TMM & 458 & \begin{tabular}{|l|}
85 \\
\end{tabular} & \begin{tabular}{|l|}
79 \\
\end{tabular} & 285 & \\
\hline Nishad & \begin{tabular}{|l} 
IDA \\
\end{tabular} & 285 & \begin{tabular}{|l}
79 \\
\end{tabular} & 85 & 458 & 743 \\
\hline Wongnrachum & $\beta$ TTM & 472 & 113 & 65 & 257 & 729 \\
\hline & \begin{tabular}{|l|} 
IDA \\
\end{tabular} & 257 & 65 & 113 & 472 & 729 \\
\hline & $\beta$ TMM & 516 & 131 & 21 & 239 & 755 \\
\hline Sehgal & IDA & 239 & 21 & 131 & 516 & 735 \\
\hline & BTM & 110 & 237 & 427 & 133 & 243 \\
\hline Pornprasert & \begin{tabular}{|l} 
IDA \\
\end{tabular} & 133 & 427 & 237 & 110 & 243 \\
\hline & $\beta$ TTM & 193 & 93 & 344 & 277 & 470 \\
\hline Sirachainan & IDA & 277 & 344 & 93 & 193 & $4 / 0$ \\
\hline Bordbar & $\beta$ TMM & 522 & 165 & 15 & 205 & 727 \\
\hline Bordbar & \begin{tabular}{|l|} 
IDA \\
\end{tabular} & 205 & 15 & 165 & 522 & 727 \\
\hline Matos and Carvalho (MC) & $\beta$ TTM & 422 & 76 & 115 & 294 & 716 \\
\hline Matos and Carvalno(MiC) & IDA & 294 & 115 & 76 & 422 & 110 \\
\hline Janel (11T) & $\beta$ TMM & 423 & 38 & 114 & 332 & 755 \\
\hline Janel (11T) & IDA & 332 & 114 & 38 & 423 & 755 \\
\hline CRUUSF & $\beta$ TMM & 413 & 102 & 124 & 268 & 682 \\
\hline CRUISE & IDA & 268 & 124 & 102 & 413 & 082 \\
\hline Index 26 स & $\beta$ TMM & 424 & 26 & 113 & 344 & 766 \\
\hline inaex 20 & IDA & 344 & 113 & 26 & 424 & 706 \\
\hline
\end{tabular}

Table 3. True positive and negative (TP and TN), false positive and negative (FP and FN) and total number of correctly identified patients $(\mathrm{TP}+\mathrm{TN})$ of each discrimination index for differential between $\beta T T(n=537)$ and IDA $(n=370)$ in patients with microcytic anemia. 


\begin{tabular}{|c|c|c|c|c|c|c|}
\hline Discriminant Formula & TPR (\%) & TNR (\%) & FNR (\%) & FPR (\%) & PPV (\%) & NPV (\%) \\
\hline England and Fraser (E\&F) & $62.94(58.70-67.04)$ & $85.41(81.39-88.84)$ & $37.06(32.96-41.30)$ & $14.59(11.16-18.61)$ & $86.22(82.41-89.48)$ & $61.36(57-65.59)$ \\
\hline $\mathrm{RBC}$ & 86.41 (83.21-89.19) & 61.94 (56.71-66.98) & $13.59(10.81-16.79)$ & $38.06(33.02-43.29)$ & $77.20(73.64-80.50)$ & $75.34(70.02-80.14)$ \\
\hline Mentzer & $89.01(86.06-91.53)$ & 78.65 (74.12-82.72) & $10.99(8.47-13.94)$ & $21.35(17.28-25.88)$ & $85.82(82.64-88.61)$ & $83.14(78.80-86.91)$ \\
\hline Srivastava & 74.86 (70.97-78.48) & $80.81(76.42-84.70)$ & $25.14(21.52-29.03)$ & $19.19(15.30-23.58)$ & $84.99(81.45-88.09)$ & $68.89(64.31-73.22)$ \\
\hline Shine and Lal (S\&L) & $100(99.32-100)$ & $17.57(13.83-21.84)$ & $0(0-0.68)$ & $82.43(78.16-86.17)$ & $63.78(60.43-67.03)$ & $100(94.48-100)$ \\
\hline Bessman & $6.33(4.42-8.72)$ & $80(75.56-83.96)$ & 93.67 (91.28-95.58) & $20(16.04-24.44)$ & $31.48(22.88-41.13)$ & $37.05(33.69-40.50)$ \\
\hline Ricerca & 98.70 (97.33-99.47) & $7.03(4.64-10.13)$ & $1.30(0.53-2.67)$ & 92.97 (89.87-95.36) & $60.64(57.31-63.90)$ & 78.79 (61.09-91.02) \\
\hline Green and King (G\&K) & $86.59(83.42-89.36)$ & $78.65(74.12-82.72)$ & $13.41(10.64-16.58)$ & $21.35(17.28-25.88)$ & $85.48(82.23-88.33)$ & $80.17(75.69-84.14)$ \\
\hline Das Gupta & $95.34(93.20-96.96)$ & $36.22(31.31-41.34)$ & $4.66(3.04-6.8)$ & $63.78(58.66-68.69)$ & $68.45(64.98-71.77)$ & $84.28(77.67-89.56)$ \\
\hline Jayabose (RDWI) & 92.55 (89.99-94.63) & $64.32(59.21-69.21)$ & $7.45(5.37-10.01)$ & $35.68(30.79-40.79)$ & $79.01(75.62-82.13)$ & $85.61(80.93-89.52)$ \\
\hline Telmissani-MCHD & $98.32(96.84-99.23)$ & $3.51(1.88-5.93)$ & $1.68(0.77-3.16)$ & $96.49(94.07-98.12)$ & $59.66(56.34-62.91)$ & $59.09(36.35-79.29)$ \\
\hline Telmissani-MDHL & $56.42(52.11-60.67)$ & $85.68(81.69-89.08)$ & 43.58 (39.33-47.89) & $14.32(10.92-18.31)$ & $85.11(80.98-88.65)$ & $57.53(53.28-61.70)$ \\
\hline Huber- Herklotz & $22.53(19.07-26.31)$ & $85.95(81.98-89.32)$ & 77.47 (73.69-80.93) & $14.05(10.68-18.02)$ & $69.94(62.52-76.67)$ & $43.32(39.70-47)$ \\
\hline Kerman I & $94.41(92.12-96.20)$ & $61.89(56.73-66.86)$ & $5.59(3.8-7.88)$ & $38.11(33.14-43.27)$ & $78.24(74.86-81.36)$ & $88.42(83.88-92.05)$ \\
\hline Kerman II & $88.64(85.65-91.20)$ & 82.16 (77.87-85.93) & $11.36(8.80-14.35)$ & $17.84(14.07-22.13)$ & $87.82(84.77-90.46)$ & $83.29(79.06-86.97)$ \\
\hline Sirdah & $80.26(76.64-83.55)$ & 88.65 (84.97-91.70) & $19.74(16.45-23.36)$ & $11.35(8.30-15.03)$ & 91.12 (88.19-93.53) & $75.58(71.25-79.55)$ \\
\hline Ehsani & 89.01 (86.06-91.53) & $81.35(77-85.19)$ & $10.99(8.47-13.94)$ & $18.65(14.81-23)$ & $87.39(84.31-90.05)$ & $83.61(79.37-87.28)$ \\
\hline Keikhaei & $88.64(85.65-91.20)$ & $72.70(67.86-77.18)$ & $11.36(8.8-14.35)$ & $27.30(22.82-32.14)$ & $82.50(79.14-85.51)$ & $81.52(76.90-85.56)$ \\
\hline Nishad & $85.29(82.01-88.18)$ & $77.03(72.40-81.22)$ & $14.71(11.82-17.99)$ & $22.97(18.78-27.60)$ & $84.35(81.01-87.30)$ & $78.30(73.70-82.42)$ \\
\hline Wongprachum & $87.90(84.83-90.53)$ & 69.46 (64.49-74.12) & $12.10(9.47-15.17)$ & $30.54(25.88-35.51)$ & $80.68(77.25-83.81)$ & 79.81 (75.01-84.06) \\
\hline Sehgal & $96.09(94.08-97.56)$ & 64.59 (59.48-69.47) & $3.91(2.44-5.92)$ & $35.41(30.53-40.52)$ & $79.75(76.45-82.78)$ & $91.92(87.92-94.93)$ \\
\hline Pornprasert & $20.48(17.15-24.15)$ & $35.95(31.05-41.07)$ & 79.52 (75.85-82.85) & $64.05(58.93-68.95)$ & $31.70(26.84-36.88)$ & $23.75(20.28-27.50)$ \\
\hline Sirachainan & $35.94(31.88-40.16)$ & $74.86(70.12-79.21)$ & 64.06 (59.84-68.12) & 25.14 (20.79-29.88) & $67.48(61.72-72.88)$ & $44.61(40.65-48.61)$ \\
\hline Bordbar & 97.21 (95.43-98.43) & $55.40(50.18-60.54)$ & $2.79(1.54-4.57)$ & $44.59(39.46-49.82)$ & 75.98 (72.61-79.13) & $93.18(89-96.13)$ \\
\hline Matos and Carvalho & $78.58(74.87-81.98)$ & $79.46(74.98-83.46)$ & $21.42(18.02-25.13)$ & $20.54(16.54-25.02)$ & $84.74(81.27-87.78)$ & $71.88(67.26-76.19)$ \\
\hline Janel $(11 \mathrm{~T})$ & $78.77(75.07-82.16)$ & $89.73(86.18-92.63)$ & $21.23(17.84-24.93)$ & $10.27(7.37-13.82)$ & 91.76 (88.86-94.10) & $74.44(70.13-78.43)$ \\
\hline CRUISE & $76.91(73.11-80.41)$ & $72.43(67.58-76.93)$ & $23.09(19.59-26.89)$ & $27.57(23.07-32.42)$ & 80.19 (76.49-83.55) & $68.37(63.51-72.95)$ \\
\hline Index26 & $78.96(75.26-82.33)$ & 92.97 (89.87-95.36) & $21.04(17.67-24.74)$ & $7.03(4.64-10.13)$ & $94.22(91.65-96.19)$ & 75.27 (71.05-79.16) \\
\hline
\end{tabular}

Table 4. Sensitivity (TPR), specificity (TNR), false positive and negative rate (FNR and FPR), positive and negative predictive values (PPV and NPV) of each discrimination index for differential $\beta T T(n=537)$ from IDA $(\mathrm{n}=370)$ in patients with microcytic anemia with their $95 \%$ exact confidence interval.

negative rate, positive and negative predictive value, Youden's index, accuracy, positive and negative likelihood ratio, diagnostic odds ratio (DOR) and area under the curve (AUC).

$$
\begin{aligned}
& \text { Sensitivity }(\text { True Positive Rate })=\frac{\text { True Positive }}{(\text { True Positive }+ \text { False Negative })} \\
& \text { Specificity (True Negative Rate) }=\frac{\text { True Negative }}{(\text { True Negative }+ \text { False Positive })}
\end{aligned}
$$$$
\text { False Negative Rate }=(1-\text { Sensitivity })
$$$$
\text { False Positive Rate }=(1-\text { Specificity })
$$$$
\text { Positive Predictive Value }(\mathrm{PPV})=\frac{\text { True Positive }}{(\text { True Positive }+ \text { False Positive })}
$$

Negative Predictive Value (NPV) $=\frac{\text { True Negative }}{(\text { True Negative }+ \text { False }}$

$$
\overline{\text { (True Negative }+ \text { False Negative) }}
$$

Youden's Index $=$ Sensitivity + Specificity -1

$$
\begin{gathered}
\text { Accuracy }=\frac{(\text { True Negative }+ \text { True Positive })}{(\text { True Negative }+ \text { True Positive }+ \text { False Positive }+ \text { False Negative })} \\
\text { PositiveLikelihood Ratio }(\mathrm{LR}+)=\frac{\text { Sensitivity }}{(1-\text { Specificity })}
\end{gathered}
$$




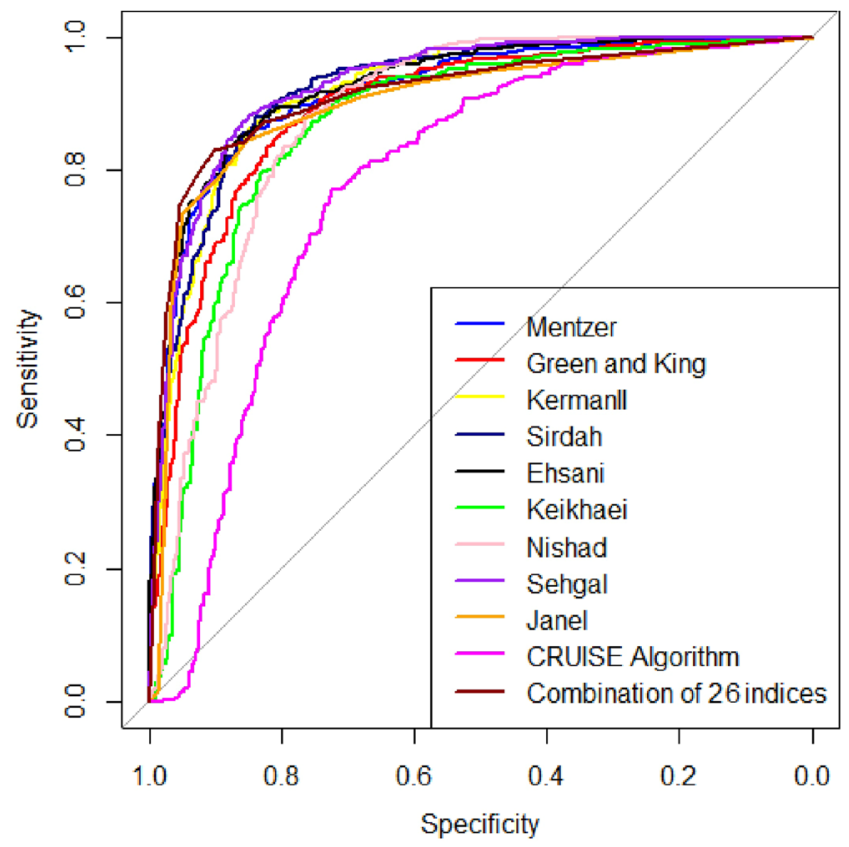

Figure 2. Reciever operating characteristic curves of discrimination indices with area under curve (AUC) higher than 0.8 (discrimination indices such as: index26, Kerman II, Ehsani, Sirdah, Janel (11T), Mentzer, Green and King (G\&K), Nishad, Keikhaei, Sehgal and CRUISE).

$$
\begin{gathered}
\text { Negative Likelihood Ratio(LR }-)=\frac{1-\text { Sensitivity }}{\text { Specificity }} \\
\text { Diagnostic Odds Ratio(DOR) }=\frac{\text { Positive Likelihood Ratio }}{\text { Negative Likelihood Ratio }}
\end{gathered}
$$

If a discrimination index had sensitivity, specificity, positive and negative predictive value, Youden's index and accuracy near to 1 , then this discrimination index has better differential performance. Discrimination index with likelihood ratio of greater than 10 , negative likelihood ratio with lower than 0.1 and high diagnostic odds ratio has a good diagnostic performance in differentiation between $\beta$ TT and IDA ${ }^{57}$. Also, receiver operating characteristic (ROC) ${ }^{58}$ curve analysis was used to calculate the AUC, and compare the amount of AUC of discrimination indices. AUC with higher value indicates an overall good performance measure for each discrimination index. A perfect diagnostic discrimination index has an AUC equal to 1. Relationship between the AUC with the diagnostic accuracy is defined as: $0.9<\mathrm{AUC}<1$ : excellent, $0.8<\mathrm{AUC}<0.9$ : very good, $0.7<\mathrm{AUC}<0.8$ : good, $0.6<$ AUC $<0.7$ : sufficient, $0.5<$ AUC $<0.6$ : bad, AUC $<0.5$ : index not useful ${ }^{57}$.

Herein, 2 new discriminating indices (CRUISE index and index26) were proposed for differentiating between $\beta$ TT and IDA. CRUISE index was created using CRUISE tree algorithm ${ }^{59,60}$, and important normalized variables were used for evaluating coefficients of hematological parameters in calculation of this index. Index 26 was created by pooling all indices except the Janel $(11 \mathrm{~T})$ index. Index 26 was computed similar to Janel $(11 \mathrm{~T})$ index $^{41}$, but index 26 was calculated by combination of 26 indices (all indices except Janel (11 T) index). Janel ( $11 \mathrm{~T}$ ) index was calculated by combining some indices (England and Fraser, RBC, Mentzer, Shine and Lal, Srivastava, Green and King, RDW, RDWI, Ricerca, Ehsani, and Sirdah). Optimum cut off for index26 was calculated using Youden's index (indeed, optimum cutoff has maximum Youden's index).

Also cluster analysis was used in order to extract homogeneous groups of discrimination indices with a similar diagnostic performance, according to stated accuracy measures for determining the each discrimination index diagnostic performance.

Cluster analysis is a technique for extracting observations homogeneous subgroups in a data set containing $\mathrm{n}$ samples and $\mathrm{P}$ predictor variables. Different algorithms are recommended for cluster analysis and some of this algorithms are known as hierarchical algorithms like single-linkage, complete-linkage, average-linkage, Ward's method, and $\mathrm{k}$-means non-hierarchical algorith $\mathrm{m}^{61}$. In this study, we proposed the cluster analysis application by using accuracy measures as predictor variables and it can be an applicable idea for determining differential indices with a similar performances. In former studies, these indices were compared only in subjective way, according to the accuracy measures like sensitivity, specificity, positive and negative predictive value, positive and negative likelihood ratio, accuracy, Youden's index and AUC 3,6,17,32,40,42,56. We used hierarchical algorithm (complete-linkage), and also the optimal number of indices subgroups with a similar performances was selected by using the package of NbClust in $\mathrm{R}$ software. This package includes 30 appropriate measures for determining the subgroups optimal number. We selected the optimal number according to the majority role. 


\begin{tabular}{|l|l|l|l|l|l|l|l|l|}
\hline Discriminant Formula & TPR & TNR & PPV & NPV & Youden's Index & Accuracy & DOR & AUC \\
\hline England and Fraser (E\&F) & 23 & 6 & 6 & 22 & 19 & 19 & 19 & 19 \\
\hline RBC & 15 & 21 & 19 & 16 & 19 & 17 & 18 & 18 \\
\hline Mentzer & 9.5 & 13 & 7 & 9 & 6 & 3 & 8 & 6 \\
\hline Srivastava & 22 & 9 & 10 & 20 & 15 & 16 & 16 & 15 \\
\hline Shine and Lal (S\&L) & 1 & 26 & 24 & 1 & 22 & 22 & & 22 \\
\hline Bessman & 28 & 10 & 28 & 27 & 27 & 27 & 26 & 27 \\
\hline Ricerca & 2 & 27 & 25 & 13 & 25 & 23 & 22 & 25 \\
\hline Green and King (G\&K) & 14 & 13 & 8 & 11 & 7 & 6 & 10 & 7 \\
\hline Das Gupta & 6 & 24 & 22 & 6 & 21 & 20 & 17 & 21 \\
\hline Jayabose (RDWI) & 8 & 20 & 17 & 5 & 13 & 12 & 11 & 13 \\
\hline Telmissani - MCHD & 3 & 28 & 26 & 23 & 26 & 24 & 23 & 26 \\
\hline Telmissani - MDHL & 24 & 5 & 9 & 24 & 20 & 21 & 21 & 20 \\
\hline Huber - Herklotz & 26 & 4 & 21 & 26 & 24 & 26 & 24 & 24 \\
\hline Kerman I & 7 & 22 & 18 & 4 & 14 & 11 & 9 & 14 \\
\hline Kerman II & 11.5 & 7 & 4 & 8 & 2 & 1 & 4 & 2 \\
\hline Sirdah & 17 & 3 & 3 & 15 & 4 & 5 & 6 & 4 \\
\hline Ehsani & 9.5 & 8 & 5 & 7 & 3 & 2 & 5 & 3 \\
\hline Keikhaei & 11.5 & 16 & 13 & 10 & 9 & 9 & 12 & 9 \\
\hline Nishad & 16 & 14 & 12 & 14 & 8 & 10 & 13 & 8 \\
\hline Wongprachum & 13 & 18 & 14 & 12 & 12 & 13 & 14 & 12 \\
\hline Sehgal & 5 & 19 & 16 & 3 & 10 & 8 & 2 & 10 \\
\hline Pornprasert & 27 & 25 & 27 & 28 & 28 & 28 & 27 & 28 \\
\hline Sirachainan & 25 & 15 & 23 & 25 & 23 & 25 & 25 & 23 \\
\hline Bordba & 4 & 23 & 20 & 2 & 16 & 14 & 3 & 16 \\
\hline Matos and Carvalho & 20 & 11 & 11 & 19 & 11 & 15 & 15 & 11 \\
\hline Janel (11 T) & 19 & 2 & 2 & 18 & 5 & 8 & 7 & 5 \\
\hline CRUISE & 21 & 17 & 15 & 21 & 17 & 18 & 20 & 18 \\
\hline Index26 & 18 & 1 & 1 & 17 & 1 & 4 & 1 & 1 \\
\hline & & & & & & & & \\
\hline
\end{tabular}

Table 5. Ranking of diagnostic performance of discrimination indices for differential $\beta T T(n=537)$ from IDA $(n=370)$ in patients with microcytic anemia based on sensitivity (TPR), specificity (TNR), positive and negative predictive values (PPV and NPV), Youden's index, accuracy, diagnostic odds ratio (DOR) and area under the curve (AUC) (lower rank shows better diagnostic performance).

Validation of the CRUISE Index and Index26. To validate the CRUISE index and index26, a cross-sectional study was performed in a referral center (Boghrat clinical center) in Tehran, Iran. A total of 6103 out-patients were screened among which 907 cases with anemia were included in this study. Classification of patients regarding having IDA or $\beta$ TT was carried out according to the WHO diagnostic criteria ${ }^{62}$. Among 907 patients with anemia, 370 of them were eligible to have IDA and 537 of them were eligible to have $\beta$ TT (Fig. 1).

Statistical analysis. Descriptive statistics such as the mean, the standard deviation (SD), the median, and interquartile range (IQR) were calculated for hematological parameters and also age variable. Mann-Whitney $\mathrm{U}$ test was used in order to compare the differences between two groups parameters ( $\beta T T$ and IDA), because of these parameters distributions were non-normal. Normality of data was evaluated by using Shapiro-Wilk test. Sex variable was tested by chi-square test for both of the $\beta$ TT and IDA groups.

Data were analyzed using a free statistical software named $\mathrm{R}$ version 5.3.0. Package epiR in $\mathrm{R}$ was used in order to calculate accuracy measures with their $95 \%$ exact confidence interval. ROC curve analysis was completed by using the package of pROC. Also, the package of OptimalCutpoints was used in order to calculate new discrimination indices cut off values by using Youden's index. Determining the clusters optimal number, or homogeneous groups of diagnostic discrimination indices with similar performances was completed by using the package of NbClust. $\mathrm{P}<0.05$ was considered significant statistical difference.

\section{Result}

537 (59\%) patients with $\beta$ TT (299 (56\%) women and 238 (44\%) men), and 370 (41\%) patients with IDA (293 (79\%) women, and $77(21 \%)$ men) were participated in this research in order to evaluate the diagnostic performance of 28 discrimination indices (two of them are new indices like CRUISE index, and index26). Chi-square test pointed out that there is significant statistical association between sex and the disease groups $\left(\chi^{2}(1)=53.41\right.$, $\mathrm{P}<0.001$ ). Hematological parameters and age variable descriptive statistics of the study groups ( $\beta$ TT and IDA) are displayed in Table 1. According to information indicated in this table, we can concluded that all variables except HCT and RDW variables present significant difference amongst the groups $(\mathrm{P}<0.001)$. 


\begin{tabular}{|c|c|c|c|c|c|}
\hline Discriminant Formula & Youden's Index (\%) & Accuracy (\%) & $\mathrm{LR}+(\%)$ & LR - (\%) & DOR (\%) \\
\hline England and Fraser (E\&F) & \begin{tabular}{|l|}
48.35 \\
$(40.09-55.88)$
\end{tabular} & $\begin{array}{l}72.11 \\
(69.06-75)\end{array}$ & $\begin{array}{l}4.31 \\
(3.34-5.56)\end{array}$ & \begin{tabular}{|l|}
0.43 \\
$(0.39-0.49)$
\end{tabular} & \begin{tabular}{|l|}
10.02 \\
$(7.092-13.93)$
\end{tabular} \\
\hline $\mathrm{RBC}$ & \begin{tabular}{|l|}
48.35 \\
$(39.92-56.17)$ \\
\end{tabular} & \begin{tabular}{|l|}
76.59 \\
$(73.68-79.32)$ \\
\end{tabular} & \begin{tabular}{|l|}
2.27 \\
$(1.98-2.60)$
\end{tabular} & $\begin{array}{l}0.22 \\
(0.17-0.28)\end{array}$ & \begin{tabular}{|l|}
10.32 \\
$(7.47-14.33)$
\end{tabular} \\
\hline Mentzer & $\begin{array}{l}67.66 \\
(60.17-74.25)\end{array}$ & \begin{tabular}{|l|}
84.78 \\
$(82.28-87.06)$
\end{tabular} & $\begin{array}{l}4.17 \\
(3.42-5.08)\end{array}$ & $\begin{array}{l}0.14 \\
(0.11-0.18)\end{array}$ & $\begin{array}{l}29.79 \\
(20.67-43.09)\end{array}$ \\
\hline Srivastava & \begin{tabular}{|l}
55.67 \\
$(47.39-63.17)$
\end{tabular} & $\begin{array}{l}77.29 \\
(74.42-79.98)\end{array}$ & \begin{tabular}{|l|}
3.90 \\
$(3.15-4.84)$
\end{tabular} & $\begin{array}{l}0.31 \\
(0.27-0.36)\end{array}$ & $\begin{array}{l}12.58 \\
(9.07-17.34)\end{array}$ \\
\hline Shine and Lal (S\&L) & $\begin{array}{l}17.57 \\
(12.80-21.83)\end{array}$ & $\begin{array}{l}66.37 \\
(63.19-69.44)\end{array}$ & $\begin{array}{l}1.21 \\
(1.16-1.27)\end{array}$ & 0 & $\infty$ \\
\hline Bessman & \begin{tabular}{|l|}
-13.67 \\
$(-20.02-7.31)$ \\
\end{tabular} & \begin{tabular}{|l|}
36.38 \\
$(33.25-39.61)$ \\
\end{tabular} & \begin{tabular}{|l|}
0.32 \\
$(0.22-0.46)$ \\
\end{tabular} & \begin{tabular}{|l|}
1.17 \\
$(1.11-1.24)$ \\
\end{tabular} & \begin{tabular}{|l|}
0.27 \\
$(0.18-0.42)$ \\
\end{tabular} \\
\hline Ricerca & \begin{tabular}{|l|l}
5.72 \\
$(1.97-9.60)$ \\
\end{tabular} & \begin{tabular}{|l|}
61.30 \\
$(58.04-64.48)$ \\
\end{tabular} & \begin{tabular}{|l|}
1.06 \\
$(1.03-1.09)$ \\
\end{tabular} & \begin{tabular}{|l|}
0.19 \\
$(0.08-0.42)$ \\
\end{tabular} & \begin{tabular}{|l|}
5.58 \\
$(2.46-13.33)$ \\
\end{tabular} \\
\hline Green and King (G\&K) & \begin{tabular}{|l|}
65.24 \\
$(57.53-72.08)$ \\
\end{tabular} & \begin{tabular}{|l|}
83.35 \\
$(80.76-85.72)$ \\
\end{tabular} & \begin{tabular}{|l|}
4.06 \\
$(3.33-4.95)$ \\
\end{tabular} & \begin{tabular}{|l}
0.17 \\
$(0.14-0.21)$ \\
\end{tabular} & \begin{tabular}{|l}
23.88 \\
$(16.74-33.80)$ \\
\end{tabular} \\
\hline Das Gupta & \begin{tabular}{|l|}
31.56 \\
$(24.52-38.31)$ \\
\end{tabular} & $\begin{array}{l}71.22 \\
(68.16-74.15) \\
\end{array}$ & \begin{tabular}{|l|}
1.49 \\
$(1.38-1.62)$ \\
\end{tabular} & $\begin{array}{l}0.13 \\
(0.09-0.19) \\
\end{array}$ & $\begin{array}{l}11.46 \\
(7.38-18.31) \\
\end{array}$ \\
\hline Jayabose (RDWI) & \begin{tabular}{|l|}
56.87 \\
$(49.20-63.83)$ \\
\end{tabular} & \begin{tabular}{|l|}
81.04 \\
$(78.33-83.54)$ \\
\end{tabular} & \begin{tabular}{|l|}
2.59 \\
$(2.26-2.98)$
\end{tabular} & $\begin{array}{l}0.12 \\
(0.09-0.16)\end{array}$ & $\begin{array}{l}21.58 \\
(15.23-32.96)\end{array}$ \\
\hline Telmissani - MCHD & $\begin{array}{l}1.83 \\
(-1.27-5.16) \\
\end{array}$ & \begin{tabular}{|l|}
59.65 \\
$(56.37-62.86)$ \\
\end{tabular} & $\begin{array}{l}1.02 \\
(1.00-1.04)\end{array}$ & $\begin{array}{l}0.48 \\
(0.21-1.10)\end{array}$ & \begin{tabular}{|l|}
2.13 \\
$(0.90-5.05)$
\end{tabular} \\
\hline Telmissani - MDHL & \begin{tabular}{|l|}
42.10 \\
$(33.80-49.75)$ \\
\end{tabular} & \begin{tabular}{|l|}
68.36 \\
$(65.22-71.37)$ \\
\end{tabular} & $\begin{array}{l}3.94 \\
(3.04-5.11)\end{array}$ & $\begin{array}{l}0.51 \\
(0.46-0.56)\end{array}$ & \begin{tabular}{|l|}
7.73 \\
$(5.53-10.85)$
\end{tabular} \\
\hline Huber - Herklotz & \begin{tabular}{|l|}
8.48 \\
$(1.05-15.63)$
\end{tabular} & \begin{tabular}{|l}
48.40 \\
$(45.10-51.71)$
\end{tabular} & $\begin{array}{l}1.60 \\
(1.19-2.16)\end{array}$ & \begin{tabular}{|l|}
0.90 \\
$(0.85-0.96)$
\end{tabular} & $\begin{array}{l}1.78 \\
(1.25-2.54)\end{array}$ \\
\hline Kerman I & $\begin{array}{l}56.30 \\
(48.85-63.06)\end{array}$ & \begin{tabular}{|l|}
81.15 \\
$(78.45-83.64)$
\end{tabular} & \begin{tabular}{|l|}
2.48 \\
$(2.17-2.83)$
\end{tabular} & $\begin{array}{l}0.09 \\
(0.06-0.13)\end{array}$ & $\begin{array}{l}27.56 \\
(17.97-41.94)\end{array}$ \\
\hline Kerman II & \begin{tabular}{|l}
70.80 \\
$(63.52-77.13)$
\end{tabular} & \begin{tabular}{|l}
86.00 \\
$(83.57-88.19)$
\end{tabular} & \begin{tabular}{|l|}
4.97 \\
$(3.98-6.20)$
\end{tabular} & $\begin{array}{l}0.14 \\
(0.11-0.18)\end{array}$ & $\begin{array}{l}35.50 \\
(24.66-52.38)\end{array}$ \\
\hline Sirdah & $\begin{array}{l}68.91 \\
(61.61-75.24)\end{array}$ & $\begin{array}{l}83.68 \\
(81.11-86.03)\end{array}$ & \begin{tabular}{|l|}
7.07 \\
$(5.30-9.43)$
\end{tabular} & \begin{tabular}{|l|}
0.22 \\
$(0.19-0.27)$
\end{tabular} & $\begin{array}{l}32.14 \\
(21.60-46.67)\end{array}$ \\
\hline Ehsani & \begin{tabular}{|l|}
70.36 \\
$(63.06-76.72)$ \\
\end{tabular} & \begin{tabular}{|l|}
85.89 \\
$(83.45-88.09)$ \\
\end{tabular} & \begin{tabular}{|l|}
4.77 \\
$(3.85-5.92)$ \\
\end{tabular} & $\begin{array}{l}0.14 \\
(0.11-0.17) \\
\end{array}$ & \begin{tabular}{|l|}
34.07 \\
$(24.26-51.49)$
\end{tabular} \\
\hline Keikhaei & \begin{tabular}{|l|}
61.34 \\
$(53.51-68.38)$ \\
\end{tabular} & \begin{tabular}{|l|}
82.14 \\
$(79.49-84.58)$ \\
\end{tabular} & \begin{tabular}{|l|}
3.25 \\
$(2.74-3.85)$ \\
\end{tabular} & $\begin{array}{l}0.16 \\
(0.12-0.20) \\
\end{array}$ & $\begin{array}{l}20.31 \\
(14.63-29.53) \\
\end{array}$ \\
\hline Nishad & \begin{tabular}{|l|}
62.32 \\
$(54.40-69.39)$ \\
\end{tabular} & \begin{tabular}{|l|}
81.92 \\
$(79.26-84.37)$ \\
\end{tabular} & \begin{tabular}{|l|}
3.71 \\
$(3.07-4.49)$ \\
\end{tabular} & $\begin{array}{l}0.19 \\
(0.15-0.24) \\
\end{array}$ & $\begin{array}{l}19.53 \\
(13.83-27.31) \\
\end{array}$ \\
\hline Wongprachum & \begin{tabular}{|l|}
57.36 \\
$(49.32-64.65)$ \\
\end{tabular} & \begin{tabular}{|l|}
80.38 \\
$(77.64-82.91)$ \\
\end{tabular} & \begin{tabular}{|l|}
2.88 \\
$(2.46-3.37)$
\end{tabular} & $\begin{array}{l}0.17 \\
(0.14-0.22)\end{array}$ & $\begin{array}{l}16.94 \\
(11.75-23.22)\end{array}$ \\
\hline Sehgal & \begin{tabular}{|l|}
60.68 \\
$(53.57-67.03)$ \\
\end{tabular} & \begin{tabular}{|l|}
83.24 \\
$(80.65-85.62)$ \\
\end{tabular} & \begin{tabular}{|l|}
2.71 \\
$(2.36-3.12)$
\end{tabular} & $\begin{array}{l}0.06 \\
(0.04-0.09)\end{array}$ & \begin{tabular}{|l|}
45.17 \\
$(27.59-72.85)$ \\
\end{tabular} \\
\hline Pornprasert & $\begin{array}{l}-43.57 \\
(-51.80--34.78)\end{array}$ & $\begin{array}{l}26.79 \\
(23.93-29.80) \\
\end{array}$ & \begin{tabular}{|l|}
0.32 \\
$(0.27-0.38)$
\end{tabular} & $\begin{array}{l}2.21 \\
(1.92-2.55)\end{array}$ & $\begin{array}{l}0.15 \\
(0.11-0.20)\end{array}$ \\
\hline Sirachainan & \begin{tabular}{|l|}
10.80 \\
$(2-19.37)$
\end{tabular} & \begin{tabular}{|l}
51.82 \\
$(48.51-55.12)$
\end{tabular} & $\begin{array}{l}1.43 \\
(1.16-1.76)\end{array}$ & $\begin{array}{l}0.86 \\
(0.78-0.93)\end{array}$ & $\begin{array}{l}1.66 \\
(1.25-2.24)\end{array}$ \\
\hline Bordbar & $\begin{array}{l}52.61 \\
(45.61-58.97)\end{array}$ & \begin{tabular}{|l|}
80.15 \\
$(77.41-82.70)$
\end{tabular} & $\begin{array}{l}2.18 \\
(1.94-2.44)\end{array}$ & \begin{tabular}{|l|}
0.05 \\
$(0.03-0.08)$
\end{tabular} & \begin{tabular}{|l|}
43.60 \\
$(24.88-75.14)$
\end{tabular} \\
\hline Matos and Carvalho & $\begin{array}{l}58.04 \\
(49.85-65.44)\end{array}$ & $\begin{array}{l}78.94 \\
(76.14-81.55)\end{array}$ & \begin{tabular}{|l|}
3.83 \\
$(3.12-4.70)$
\end{tabular} & \begin{tabular}{|l|}
0.27 \\
$(0.23-0.32)$
\end{tabular} & $\begin{array}{l}14.20 \\
(10.25-19.66)\end{array}$ \\
\hline Janel (11T) & $\begin{array}{l}68.50 \\
(61.24-74.79)\end{array}$ & \begin{tabular}{|l}
83.24 \\
$(80.65-85.62)$
\end{tabular} & \begin{tabular}{|l|}
7.67 \\
$(5.66-$ \\
$10.40)$ \\
\end{tabular} & $\begin{array}{l}0.24 \\
(0.20-0.28)\end{array}$ & $\begin{array}{l}31.96 \\
(21.86-48.09)\end{array}$ \\
\hline CRUISE & $\begin{array}{l}49.34 \\
(40.69-57.33)\end{array}$ & \begin{tabular}{|l|}
75.08 \\
$(72.13-77.87)$
\end{tabular} & $\begin{array}{l}2.79 \\
(2.35-3.31)\end{array}$ & $\begin{array}{l}0.32 \\
(0.27-0.38)\end{array}$ & \begin{tabular}{|l|}
8.72 \\
$(6.46-11.86)$
\end{tabular} \\
\hline Index26 & \begin{tabular}{|l}
71.93 \\
$(65.13-77.69)$
\end{tabular} & $\begin{array}{l}84.67 \\
(82.16-86.96)\end{array}$ & $\begin{array}{l}11.24 \\
(7.74- \\
16.32)\end{array}$ & $\begin{array}{l}0.23 \\
(0.19-0.27)\end{array}$ & $\begin{array}{l}48.87 \\
(31.67-77.81)\end{array}$ \\
\hline
\end{tabular}

Table 6. Youden's index, accuracy, positive and negative likelihood ratio ( $\mathrm{LR}+$ and $\mathrm{LR}-$ ) and diagnostic odds ratio (DOR) of each discrimination index for differential $\beta T T(n=537)$ from IDA $(n=370)$ in patients with microcytic anemia with their $95 \%$ exact confidence interval.

Discrimination indices with their cut off are shown in Table 2. The number of true positive and negative, false positive and negative, and total number of correctly identified patients (true positive + true negative) are displayed in Table 3 for each discrimination index. Table 4 indicates sensitivity, specificity, false positive and negative rate, and positive and negative predictive values for 28 discrimination indices, and also in Table 5 the rank of these discrimination indices according to accuracy measures is shown.

Table 4 represents that none of discrimination indices have $100 \%$ specificity and $100 \%$ positive predictive value. Also, none of indices except Shine and Lal (S\&L) have $100 \%$ sensitivity and $100 \%$ negative predictive value, 


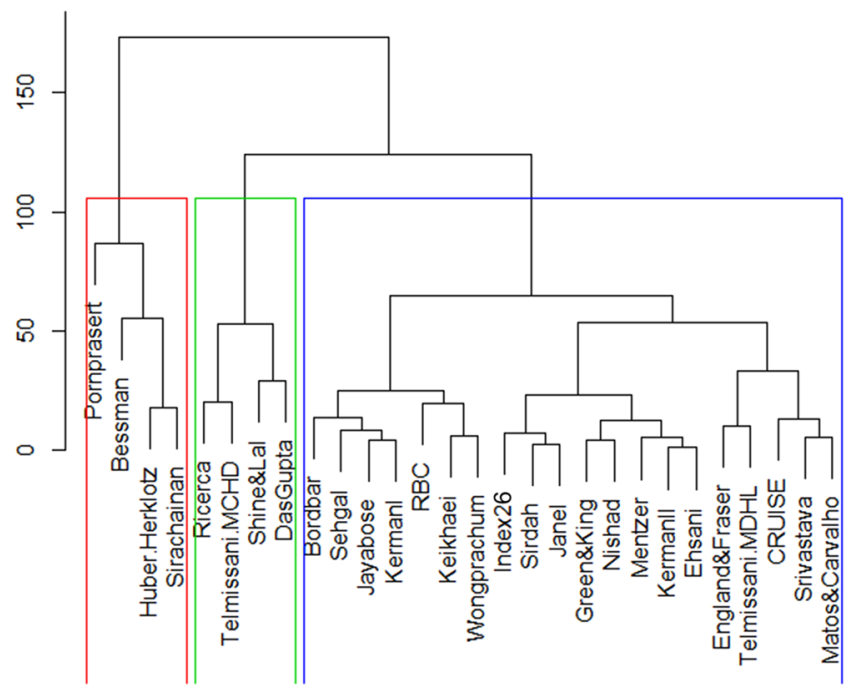

Figure 3. Dendrogram from cluster analysis for extracting homogeneous groups of diagnostic discrimination indices with similar performance (each rectangles includes diagnostic discrimination indices with similar performance).

but this index has very high false positive rate. According to information indicated in the Table 4 and the Table 5, Shine and Lal (S\&L) and Bessman point out the highest and lowest sensitivity (the lowest and highest false negative rate) in 3 TT diagnose, respectively, and index 26 and Telmissani-MCHD index indicate the highest and lowest specificity (the lowest and highest false positive rate) in IDA diagnose, respectively. Also index26 and Bessman showed the highest and lowest positive predictive value, respectively, and Shine and Lal (S\&L) and Pornprasert had highest and lowest negative predictive value (Table 4 and Table 5).

Table 5 and Table 6 presented that lowest Youden's index is related to the Pornprasert, and the highest amount is related to the index26. Also, these tables show that KermanII and Pornprasert have the highest and lowest accuracy, respectively, and the highest DOR is belong to index 26 , and the lowest is belong to Pornprasert. Two new indices introduced earlier (CRUISE index and index26), have better performance than some of the discrimination indices, which were listed in Table 2 (Table 5). Due to the findings, none of indices have LR $+>10$, and only KermanI index has LR $-<0.1$.

Each discrimination index AUC is shown in Table 7. Also, Fig. 2 showed the ROC curves for discrimination formula with the amount of AUC higher than 0.8 (Kerman II, Ehsani, Sirdah, Janel (11 T), Mentzer, Green and King (G\&K), Nishad, Keikhaei and Sehgal), and two new indices (CRUISE index and index26). Indices with the amount of AUC higher than 0.8 have very appropriate diagnostic accuracy in the discrimination between $\beta$ TT and IDA, and also CRUISE index has good diagnostic accuracy. AUC of all indices except Telmissani-MCHD were statistically significant, in regard to the amount of AUC equal to $0.5(\mathrm{P}<0.001)$ (Table 7), and AUC of Bessman and Pornprasert were significantly less than $0.5(\mathrm{P}<0.001)$. As shown in Tables 5 and 7 , the highest AUC is related to index26, and the lowest AUC is related to the Pornprasert index. Comparison between AUCs of discrimination formula (indices with AUC higher than 0.8), and two new indices are displayed in Table 8. There was a significant difference between AUC of CRUISE index and other indices, which the AUC of this index was significantly less than other indices $(\mathrm{P}<0.001)$ (Table 8), but this index has higher AUC than the amount of other indices recorded in Table 2 (Table 7). Table 8 also represented that the AUC of index26 is significantly higher than Green and King (G\&K), Keikhaei, Nishad, Sehgal, Janel (11 T) and CRUISE index $(\mathrm{P}<0.05)$, but there is no significant difference between AUC of this index and other indices like Mentzer, Kerman II, Ehsani and Sirdah (P >0.05).

Cluster analysis dendrogram (this plot represents steps in the cluster analysis) is presented in Fig. 3. Cluster analysis extracted three homogenous groups. First one of them includes discrimination indices like Pornprasert, Bessman, Huber -Herklotz, and Sirachainan. Second group includes Ricerca, Telmissani-MCHD, Shine and Lal (S\&L), Das Gupta, and the third group includes discrimination indices like Bordbar, Sehgal, Jayabose, KermanI, RBC, Keikhaei, Wongprachum, Index26, Sirdah, Janel (11 T), Green and King (G\&K), Nishad, Mentzer, KermanII, Ehsani, England and Fraser (E\&F), Telmissani-MDHL, Srivastava, CRUISE. So two new introduced indices in this study have similar performances to indices of third homogenous group.

\section{Discussion}

$\beta$ TT and IDA are known as common causes for microcytic anemia, and these two hematologic disorders typically have similar clinical and experimental conditions. The definitive diagnostic method for the $\beta$ TT is based on the $\mathrm{HbA} 2$ increase $\mathrm{e}^{17,18}$, and the principal methods for diagnosis of IDA based on the increase in TIBC, as same as a decrease in serum iron, serum ferritin, and transferrin saturation ${ }^{9}$.

The exact discrimination between these two hematologic disorders is very vital, because the correct treatment and its proper diagnosis through premarital genetic counseling, would prevent the attendant risk of thalassemia major child birth. Considering the importance of differentiating between $\beta$ TT and IDA, several different indices 


\begin{tabular}{|l|l|l|l|l|}
\hline Discriminant Formula & AUC & SE & 95\% CI & p-value \\
\hline England and Fraser (E\&F) & 0.742 & 0.0139 & $0.714-0.769$ & $<0.001$ \\
\hline RBC & 0.747 & 0.0146 & $0.718-0.775$ & $<0.001$ \\
\hline Mentzer & 0.838 & 0.0126 & $0.814-0.863$ & $<0.001$ \\
\hline Srivastava & 0.778 & 0.0139 & $0.751-0.806$ & $<0.001$ \\
\hline Shine and Lal (S\&L) & 0.588 & 0.0099 & $0.568-0.607$ & $<0.001$ \\
\hline Bessman & 0.432 & 0.0117 & $0.409-0.455$ & $<0.001$ \\
\hline Ricerca & 0.529 & 0.0071 & $0.515-0.542$ & $<0.001$ \\
\hline Green and King (G\&K) & 0.826 & 0.0130 & $0.801-0.852$ & $<0.001$ \\
\hline Das Gupta & 0.658 & 0.0133 & $0.632-0.684$ & $<0.001$ \\
\hline Jayabose (RDWI) & 0.784 & 0.0137 & $0.757-0.811$ & $<0.001$ \\
\hline Telmissani - MCHD & 0.509 & 0.0055 & $0.498-0.520$ & 0.0970 \\
\hline Telmissani - MDHL & 0.711 & 0.0141 & $0.683-0.738$ & $<0.001$ \\
\hline Huber - Herklotz & 0.542 & 0.0128 & $0.517-0.567$ & 0.001 \\
\hline Kerman I & 0.782 & 0.0136 & $0.755-0.808$ & $<0.001$ \\
\hline Kerman II & 0.854 & 0.0121 & $0.830-0.878$ & $<0.001$ \\
\hline Sirdah & 0.845 & 0.0119 & $0.821-0.868$ & $<0.001$ \\
\hline Ehsani & 0.852 & 0.0122 & $0.828-0.876$ & $<0.001$ \\
\hline Keikhaei & 0.807 & 0.0135 & $0.780-0.833$ & $<0.001$ \\
\hline Nishad & 0.812 & 0.0134 & $0.785-0.838$ & $<0.001$ \\
\hline Wongprachum & 0.787 & 0.0139 & $0.759-0.814$ & $<0.001$ \\
\hline Sehgal & 0.803 & 0.0131 & $0.778-0.829$ & $<0.001$ \\
\hline Pornprasert & 0.282 & 0.018 & $0.247-0.317$ & $<0.001$ \\
\hline Sirachainan & 0.554 & 0.0153 & $0.524-0.584$ & 0.0004 \\
\hline Bordbar & 0.763 & 0.0134 & $0.737-0.789$ & $<0.001$ \\
\hline Matos and Carvalho & 0.790 & 0.0138 & $0.763-0.817$ & $<0.001$ \\
\hline Janel (11T) & 0.843 & 0.0119 & $0.819-0.866$ & $<0.001$ \\
\hline CRUISE & 0.747 & 0.0148 & $0.718-0.776$ & $<0.001$ \\
\hline Index26 & 0.858 & 0.0111 & $0.836-0.879$ & $<0.001$ \\
\hline & & & & \\
\hline
\end{tabular}

Table 7. Area under the curve (AUC) of each discrimination index for differential $\beta T T(n=537)$ from IDA $(\mathrm{n}=370)$ in patients with microcytic anemia with their $95 \%$ confidence interval (SE: Standard Error, CI: Confidence Interval).

have been proposed in large-scale researches; additionally, these indices showed different diagnostic performance, and none of these indices had definitive diagnosis in various studies.

It is possible to discriminate between $\beta$ TT and IDA without using expensive tests with high performance index. We presented two new discriminating indices between these two common microcytic anemia, and also compared these two indicators performance with 26 different published indices. This study findings indicated that none of the discriminating indices provided $100 \%$ sensitivity and specificity. Consequently, the Shine and Lal index showed a sensitivity and a negative predictive value, but with respect to the AUC, it had a poor performance in the differentiation between the 3 TT and IDA. It is important to remember that this index has expressed as the best discriminating index for differentiation between $\beta$ TT and IDA in former researches ${ }^{[9,50,63}$. Shen et al., reported that S \& L index had a low AUC as same as this study ${ }^{55}$. In the present study, index 26 had $100 \%$ specificity and complete positive predictive value. In addition, according to Youden's index, DOR, and AUC, this index is a differential index with superior performance for differentiation between the $\beta T T$ and IDA. Accuracy measure like Youden's index, accuracy, DOR, and AUC take both sensitivity and specificity into consideration, so they can present the discrimination indices performance more accurately than other criteria. According to these criteria and also Table 6, index 26 indicates better performance in comparison to the other discrimination indices.

Also, by comparing the AUCs of various discriminating indices, this test performance was better than the differential indices significantly, like Green and King, Keikhaei, Nishad, Sehgal and Janel (11T). Considering the worth of index 26 in this study, this index is still difficult to calculate, and we are developing a calculator-based approach on differential indices expressed in the results, and in the future works we will introduce this protocol, in order to solve this problem. By using this calculator, we can determine the accuracy and each indicator outcome easily and quickly. Thus, it can be concluded that the differential indices, including Mentzer, Kerman II, Ehsani, Sirdah, janel (11 T) and index 26 are reliable indices for discrimination between the 3 TT and IDA. Another recommended index was CRUISE, which showed a good diagnostic performance, but its AUC was significantly lower compared to the other indices with the very appropriate diagnostic performance (AUC $>0.8$ ). As a result, this index has a superior performance compared to some of before stated indices. Several studies proposed new discrimination indices by using discriminant analysis for differentiating between the $\beta$ TT and IDA (these indices are Nishad, Matos and Carvalho, Sirachainan and Das Gupta $)^{27,35,39,64,65}$. We used CRUISE tree algorithm for recommending a new discrimination index, because tree-based methods are non-parametric methods, and these methods have some advantages over the traditional statistical methods like discriminant analysis. Some of these advantages are known as following: without needing to 


\begin{tabular}{|c|c|c|c|c|c|c|c|c|c|c|}
\hline & G\&K & Mentzer & Kerman II & Sirdah & Ehsani & Keikhaei & Nishad & Sehgal & Janel (11 T) & CRUISE \\
\hline Mentzer & $\begin{array}{l}\mathrm{AUC}_{\mathrm{d}}=0.012 \\
\mathrm{SE}=0.0145 \\
\mathrm{P}=0.404\end{array}$ & & & & & & & & & \\
\hline Kerman II & $\begin{array}{l}\mathrm{AUC}_{\mathrm{d}}=0.028 \\
\mathrm{SE}=0.0156 \\
\mathrm{P}=0.074\end{array}$ & $\begin{array}{l}\mathrm{AUC}_{\mathrm{d}}=0.016 \\
\mathrm{SE}=0.009 \\
\mathrm{P}=0.0810\end{array}$ & & & & & & & & \\
\hline Sirdah & $\begin{array}{l}\mathrm{AUC}_{\mathrm{d}}=0.018 \\
\mathrm{SE}=0.0125 \\
\mathrm{P}=0.142\end{array}$ & $\begin{array}{l}\mathrm{AUC}_{\mathrm{d}}=0.006 \\
\mathrm{SE}=0.0111 \\
\mathrm{P}=0.575\end{array}$ & $\begin{array}{l}\mathrm{AUC}_{\mathrm{d}}=-0.009 \\
\mathrm{SE}=0.0125 \\
\mathrm{P}=0.450\end{array}$ & & & & & & & \\
\hline Ehsani & $\begin{array}{l}\mathrm{AUC}_{\mathrm{d}}=0.026 \\
\mathrm{SE}=0.015 \\
\mathrm{P}=0.089\end{array}$ & $\begin{array}{l}\mathrm{AUC}_{\mathrm{d}}=0.013 \\
\mathrm{SE}=0.0057 \\
\mathrm{P}=0.017\end{array}$ & $\begin{array}{l}\mathrm{AUC}_{\mathrm{d}}=-0.002 \\
\mathrm{SE}=0.0073 \\
\mathrm{P}=0.763\end{array}$ & $\begin{array}{l}\mathrm{AUC}_{\mathrm{d}}=0.007 \\
\mathrm{SE}=0.0114 \\
\mathrm{P}=0.524\end{array}$ & & & & & & \\
\hline Keikhaei & $\begin{array}{l}\mathrm{AUC}_{\mathrm{d}}=-0.019 \\
\mathrm{SE}=0.0094 \\
\mathrm{P}=0.039\end{array}$ & $\begin{array}{l}\mathrm{AUC}_{\mathrm{d}=}-0.0316 \\
\mathrm{SE}=0.0136 \\
\mathrm{P}=0.02\end{array}$ & $\begin{array}{l}\mathrm{AUC}_{\mathrm{d}}=-0.047 \\
\mathrm{SE}=0.0146 \\
\mathrm{P}=0.001\end{array}$ & $\begin{array}{l}\mathrm{AUC}_{\mathrm{d}}=-0.038 \\
\mathrm{SE}=0.0134 \\
\mathrm{P}=0.005\end{array}$ & $\begin{array}{l}\mathrm{AUC}_{\mathrm{d}}=-0.045 \\
\mathrm{SE}=0.0142 \\
\mathrm{P}=0.001\end{array}$ & & & & & \\
\hline Nishad & $\begin{array}{l}\text { AUC }_{d}=-0.015 \\
S E=0.0183 \\
P=0.425\end{array}$ & $\begin{array}{l}\mathrm{AUC}_{\mathrm{d}}=-0.027 \\
\mathrm{SE}=0.0141 \\
\mathrm{P}=0.057\end{array}$ & $\begin{array}{l}\mathrm{AUC}_{\mathrm{d}}=-0.042 \\
\mathrm{SE}=0.0119 \\
\mathrm{P}=0.0004\end{array}$ & $\begin{array}{l}\mathrm{AUC}_{\mathrm{d}}=-0.033 \\
\mathrm{SE}=0.0161 \\
\mathrm{P}=0.0411\end{array}$ & $\begin{array}{l}\mathrm{AUC}_{\mathrm{d}}=-0.040 \\
\mathrm{SE}=0.0131 \\
\mathrm{P}=0.002\end{array}$ & $\begin{array}{l}\mathrm{AUC}_{\mathrm{d}}=0.005 \\
\mathrm{SE}=0.0181 \\
\mathrm{P}=0.788\end{array}$ & & & & \\
\hline Sehgal & $\begin{array}{l}\mathrm{AUC}_{\mathrm{d}}=-0.023 \\
\mathrm{SE}=0.017 \\
\mathrm{P}=0.18\end{array}$ & $\begin{array}{l}\mathrm{AUC}_{\mathrm{d}}=-0.035 \\
\mathrm{SE}=0.0116 \\
\mathrm{P}=0.003\end{array}$ & $\begin{array}{l}\mathrm{AUC}_{\mathrm{d}}=-0.051 \\
\mathrm{SE}=0.012 \\
\mathrm{P}<0.001\end{array}$ & $\begin{array}{l}\mathrm{AUC}_{\mathrm{d}}=-0.041 \\
\mathrm{SE}=0.0149 \\
\mathrm{P}=0.006\end{array}$ & $\begin{array}{l}\mathrm{AUC}_{\mathrm{d}}=-0.048 \\
\mathrm{SE}=0.0112 \\
\mathrm{P}<0.001\end{array}$ & $\begin{array}{l}\mathrm{AUC}_{\mathrm{d}}=-0.003 \\
\mathrm{SE}=0.0165 \\
\mathrm{P}=0.841\end{array}$ & $\begin{array}{l}\mathrm{AUC}_{\mathrm{d}}=-0.008 \\
\mathrm{SE}=0.0124 \\
\mathrm{P}=0.51\end{array}$ & & & \\
\hline Janel (11 T) & $\begin{array}{l}\mathrm{AUC}_{\mathrm{d}}=0.0163 \\
\mathrm{SE}=0.012 \\
\mathrm{P}=0.176\end{array}$ & $\begin{array}{l}\mathrm{AUC}_{\mathrm{d}}=0.004 \\
\mathrm{SE}=0.0111 \\
\mathrm{P}=0.707\end{array}$ & $\begin{array}{l}\mathrm{AUC}_{\mathrm{d}}=-0.011 \\
\mathrm{SE}=0.0124 \\
\mathrm{P}=0.355\end{array}$ & $\begin{array}{l}\mathrm{AUC}_{\mathrm{d}}=-0.002 \\
\mathrm{SE}=0.0061 \\
\mathrm{P}=0.738\end{array}$ & $\begin{array}{l}\mathrm{AUC}_{\mathrm{d}=}-0.009 \\
\mathrm{SE}=0.0115 \\
\mathrm{P}=0.416\end{array}$ & $\begin{array}{l}\mathrm{AUC}_{\mathrm{d}}=0.036 \\
\mathrm{SE}=0.0123 \\
\mathrm{P}=0.004\end{array}$ & $\begin{array}{l}\text { AUC }_{d}=0.031 \\
S E=0.0162 \\
P=0.057\end{array}$ & $\begin{array}{l}\mathrm{AUC}_{\mathrm{d}}=0.039 \\
\mathrm{SE}=0.0148 \\
\mathrm{P}=0.008\end{array}$ & & \\
\hline CRUISE & $\begin{array}{l}\mathrm{AUC}_{\mathrm{d}}=-0.08 \\
\mathrm{SE}=0.0166 \\
\mathrm{P}<0.001\end{array}$ & $\begin{array}{l}\mathrm{AUC}_{\mathrm{d}}=-0.092 \\
\mathrm{SE}=0.0184 \\
\mathrm{P}<0.001\end{array}$ & $\begin{array}{l}\mathrm{AUC}_{\mathrm{d}}=-0.107 \\
\mathrm{SE}=0.0186 \\
\mathrm{P}<0.001\end{array}$ & $\begin{array}{l}\mathrm{AUC}_{\mathrm{d}}=-0.098 \\
\mathrm{SE}=0.0167 \\
\mathrm{P}<0.001\end{array}$ & $\begin{array}{l}\mathrm{AUC}_{\mathrm{d}}=-0.105 \\
\mathrm{SE}=0.0185 \\
\mathrm{P}<0.001\end{array}$ & $\begin{array}{l}\mathrm{AUC}_{\mathrm{d}}=-0.06 \\
\mathrm{SE}=0.0178 \\
\mathrm{P}=0.0008\end{array}$ & $\begin{array}{l}\mathrm{AUC}_{\mathrm{d}}=-0.065 \\
\mathrm{SE}=0.0209 \\
\mathrm{P}=0.0019\end{array}$ & $\begin{array}{l}\mathrm{AUC}_{\mathrm{d}}=-0.057 \\
\mathrm{SE}=0.0191 \\
\mathrm{P}=0.0029\end{array}$ & $\begin{array}{l}\mathrm{AUC}_{\mathrm{d}}=-0.096 \\
\mathrm{SE}=0.0172 \\
\mathrm{P}<0.001\end{array}$ & \\
\hline Index 26 & $\begin{array}{l}\mathrm{AUC}_{\mathrm{d}}=0.033 \\
\mathrm{SE}=0.0125 \\
\mathrm{P}=0.0076\end{array}$ & $\begin{array}{l}\mathrm{AUC}_{\mathrm{d}}=0.021 \\
\mathrm{SE}=0.0112 \\
\mathrm{P}=0.0566\end{array}$ & $\begin{array}{l}\mathrm{AUC}_{\mathrm{d}}=0.006 \\
\mathrm{SE}=0.0115 \\
\mathrm{P}=0.6231\end{array}$ & $\begin{array}{l}\mathrm{AUC}_{\mathrm{d}}=0.015 \\
\mathrm{SE}=0.008 \\
\mathrm{P}=0.0627\end{array}$ & $\begin{array}{l}\mathrm{AUC}_{\mathrm{d}}=0.008 \\
\mathrm{SE}=0.0107 \\
\mathrm{P}=0.4625\end{array}$ & $\begin{array}{l}\mathrm{AUC}_{\mathrm{d}}=0.053 \\
\mathrm{SE}=0.0124 \\
\mathrm{P}<0.001\end{array}$ & $\begin{array}{l}\mathrm{AUC}_{\mathrm{d}}=0.048 \\
\mathrm{SE}=0.0153 \\
\mathrm{P}=0.0017\end{array}$ & $\begin{array}{l}\mathrm{AUC}_{\mathrm{d}}=0.056 \\
\mathrm{SE}=0.0143 \\
\mathrm{P}=0.0001\end{array}$ & $\begin{array}{l}\mathrm{AUC}_{\mathrm{d}}=0.017 \\
\mathrm{SE}=0.006 \\
\mathrm{P}=0.0044\end{array}$ & $\begin{array}{l}\mathrm{AUC}_{\mathrm{d}}=0.113 \\
\mathrm{SE}=0.0177 \\
\mathrm{P}<0.001\end{array}$ \\
\hline
\end{tabular}

Table 8. Comparison between area under the curve (AUC) values of discrimination indices with AUC higher than 0.8 for differential $\beta$ TT $(n=537)$ from IDA $(n=370)$ in patients with microcytic anemia $\left(A U C_{d}=A_{U} C_{\text {row }}\right.$ - $\mathrm{AUC}_{\text {column }}$, SE: Standard Error $\left(\mathrm{AUC}_{\mathrm{d}}\right)$ ).

determine assumptions about the functional form between outcome variable and predictor variables, useful for dealing with nonlinear relationships and high-order interactions, and robust to outliers and multicollinearity. In this study, CRUISE index showed a high AUC in comparison with the Sirachainan and Das Gupta indices.

Different studies are conducted in order to assess the differential indices diagnostic performance for discriminating between the $\beta$ TT and IDA in different populations. Also, these studies indicated different results. We mention index with best diagnostic performance based on the highest AUC or Youden's index here in some conducted studies in different populations.

Iranian population: Ghafouri et al. in $2006^{46}$ : Mentzer index, Rahim and Keikhaei in $2009^{45}$ : Shine and Lal index in patients $<10$ years and RDW and RDWI index in patients with the age of 10 to 57 years old, Ehsani et al. in 200933: Mentzer index and Ehsani index, Ahmadi et al. in 200944: Shine and Lal index, Keikhaei in $2010^{34}$ : Keikhaei index, Sargolzaie and Miri-Moghaddam in 2014 ${ }^{53}$ : Green and King index, Bordbar et al. in $2015^{40}$ : Bordbar index. Thailand population: Sirachainan et al. in 2014 ${ }^{39}$ : Sirachainan index. Indian population: Tripathi et al. in $2015^{66}$ : Mentzer index, Piplani et al. in $2016^{67}$ : Mentzer index. Turkey population: Demir et al. in 2002 ${ }^{17}$ : RBC index, Beyan et al. in 2007 RBC index, Vehapoglu et al. 2014 $4^{56}$ : Mentzer index. Italy population: Ferrara et al. in $2010^{68}$ : England and Fraser index. Kuwait population: AlFadhli et al. in $2006^{49}$ : England and Fraser index. Sri Lanka population: Nishad et al. in 2012 $2^{35}$ : Nishad index. Palestinian population: Sirdah et al. in $2007^{32}$ : Sirdah index. Brazilian population: Matos et al. in 2013 ${ }^{54}$. Green and King index. Chinese population: Shen et al. in 2010 55 : Green and King index. France population: Janel et al. in $2011^{41}: 11$ T, Green and King, RDWI and Sirdah index. Saudi Arabia population: Jameel et al. in $2017^{69}$ : RDWI index.

Conclusion and future directions. This cross-sectional study was conducted on Iranian patients diagnosed to have $\beta$ TT and IDA. In this study, two new discriminating indices were proposed for differentiating between the $\beta$ TT and IDA, and these indices presented a relatively similar diagnostic performance according to cluster analysis compared to different indices reported in the literature. Index 26 indicated better performance in comparison with the other discriminating indices. This low-cost index can be useful for differentiating between the $\beta$ TT and IDA, thus using this index, costs for health system can be minimized in regions with limited financial resources. Also, study results showed that data mining methods like tree-based classification models can be used in order to recommend new discriminating indices for differentiating between the $\beta T$ T and IDA. CRUISE index was found to have a superior performance compared to some of discriminating indices. This study was also the first study in which cluster analysis was applied for identifying homogeneous subgroups of discriminating indices with similar diagnostic function. Accordingly, it is recommended to use cluster analysis for determining discriminating indices with similar diagnostic performance for future studies.

Received: 12 December 2018; Accepted: 15 November 2019;

Published online: 09 December 2019 


\section{References}

1. Kara, B., Çal, S., Aydogan, A. \& Sarper, N. The prevalence of anemia in adolescents: a study from Turkey. Journal of Pediatric Hematology/Oncology 28, 316-321 (2006).

2. Brittenham, G. Disorders of iron metabolism: iron deficiency and overload. Hematology: basic principles and practice (2000).

3. Rathod, D. A. et al. Usefulness of cell counter-based parameters and formulas in detection of $\beta$-thalassemia trait in areas of high prevalence. American Journal of Clinical Pathology 128, 585-589 (2007).

4. Angastiniotis, M. \& Modell, B. Global epidemiology of hemoglobin disorders. Annals of the New York Academy of Sciences 850, 251-269 (1998).

5. Weatherall, D. \& Clegg, J. B. Inherited haemoglobin disorders: an increasing global health problem. Bulletin of the World Health Organization 79, 704-712 (2001).

6. Urrechaga, E., Borque, L. \& Escanero, J. F. The role of automated measurement of RBC subpopulations in differential diagnosis of microcytic anemia and $\beta$-thalassemia screening. American Journal of Clinical Pathology 135, 374-379 (2011).

7. Galanello, R. \& Origa, R. Beta-thalassemia. Orphanet journal of rare diseases 5, 11 (2010).

8. Hallberg, L. Iron requirements. Biological trace element research 35, 25-45 (1992).

9. Lafferty, J. D., Crowther, M. A., Ali, M. A. \& Levine, M. The evaluation of various mathematical RBC indices and their efficacy in discriminating between thalassemic and non-thalassemic microcytosis. American Journal of Clinical Pathology 106, 201-205 (1996).

10. Bessman, J. D., Gilmer, P. R. \& Gardner, F. H. Improved classification of anemias by MCV and RDW. American Journal of Clinical Pathology 80, 322-326 (1983).

11. Yang, Z., Chaffin, C. H., Easley, P. L., Thigpen, B. \& Reddy, V. V. Prevalence of elevated hemoglobin A2 measured by the CAPILLARYS system. American Journal of Clinical Pathology 131, 42-48 (2009).

12. Cao, A., Rosatelli, M. C., Monni, G. \& Galanello, R. Screening for thalassemia: a model of success. Obstetrics and Gynecology. Clinics 29, 305-328 (2002).

13. Kiss, T. L., Ali, M. A., Levine, M. \& Lafferty, J. D. An algorithm to aid in the investigation of thalassemia trait in multicultural populations. Archives of pathology \& laboratory medicine 124, 1320-1323 (2000).

14. Thomas, C. \& Thomas, L. Biochemical markers and hematologic indices in the diagnosis of functional iron deficiency. Clinical chemistry 48, 1066-1076 (2002)

15. Goddard, A. F., James, M. W., McIntyre, A. S. \& Scott, B. B. Guidelines for the management of iron deficiency anaemia. Gut, gut. 2010, 228874 (2011).

16. Mosca, A., Paleari, R., Ivaldi, G., Galanello, R. \& Giordano, P. The role of haemoglobin A2 testing in the diagnosis of thalassaemias and related haemoglobinopathies. Journal of Clinical Pathology 62, 13-17 (2009).

17. Demir, A., Yarali, N., Fisgin, T., Duru, F. \& Kara, A. Most reliable indices in differentiation between thalassemia trait and iron deficiency anemia. Pediatrics International 44, 612-616 (2002).

18. Oliveri, N. The beta-thalassemias. N Engl J Med 341, 99-109 (1999).

19. England, J. \& Fraser, P. Differentiation of iron deficiency from thalassaemia trait by routine blood-count. The Lancet 301, 449-452 (1973).

20. Klee, G. G., Fairbanks, V. F., Pierre, R. V. \& O'sullivan, M. B. Routine erythrocyte measurements in diagnosis of iron-deficiency anemia and thalassemia minor. American Journal of Clinical Pathology 66, 870-877 (1976)

21. Mentzer, W. Differentiation of iron deficiency from thalassaemia trait. The Lancet 301, 882 (1973).

22. Srivastava, P. \& Bevington, J. Iron deficiency and/or Thalassaemia trait. The Lancet 301, 832 (1973).

23. Shine, I. \& Lal, S. A strategy to detect $\beta$-thalassaemia minor. The Lancet 309, 692-694 (1977).

24. Bessman, J. D. \& Feinstein, D. Quantitative anisocytosis as a discriminant between iron deficiency and thalassemia minor. Blood 53, 288-293 (1979).

25. Ricerca, B. et al. Differentiation of iron deficiency from thalassaemia trait: a new approach. Haematologica 72, 409-413 (1986).

26. Green, R. \& King, R. A new red cell discriminant incorporating volume dispersion for differentiating iron deficiency anemia from thalassemia minor. Blood cells 15, 481-495 (1989).

27. Gupta, A. D., Hegde, C. \& Mistri, R. Red cell distribution width as a measure of severity of iron deficiency in iron deficiency anemia. Indian J Med Res 100, 177-183 (1994).

28. Jayabose, S. et al. \# 262 Differentiating iron deficiency anemia from thalassemia minor by using an RDW-based index. Journal of Pediatric Hematology/Oncology 21, 314 (1999).

29. Telmissani, O. A., Khalil, S. \& Roberts, G. T. Mean density of hemoglobin per liter of blood: a new hematologic parameter with an inherent discriminant function. Laboratory Hematology 5, 149-152 (1999).

30. Huber, A. R. et al. In Schweiz Med Forum. 947-952 (2004).

31. Kohan, N. \& Ramzi, M. Evaluation of sensitivity and specificity of Kerman index I and II in screening beta thalassemia minor. (2008).

32. Sirdah, M., Tarazi, I., Al Najjar, E. \& Al Haddad, R. Evaluation of the diagnostic reliability of different RBC indices and formulas in the differentiation of the $\beta$-thalassaemia minor from iron deficiency in Palestinian population. International Journal of Laboratory Hematology 30, 324-330 (2008).

33. Ehsani, M., Shahgholi, E., Rahiminejad, M., Seighali, F. \& Rashidi, A. A new index for discrimination between iron deficiency anemia and beta-thalassemia minor: results in 284 patients. Pakistan journal of biological sciences: PJBS 12, 473-475 (2009).

34. Keikhaei, B. A new valid formula in differentiating iron deficiency anemia from ß-thalassemia trait. Pakist J Med Sci 26, 368-373 (2010).

35. Nishad, A. A. N., Pathmeswaran, A., Wickremasinghe, A. \& Premawardhena, A. The Thal-index with the BTT prediction. exe to discriminate ß-thalassaemia traits from other microcytic anaemias. (2012).

36. Wongprachum, K. et al. Proxy indicators for identifying iron deficiency among anemic vegetarians in an area prevalent for thalassemia and hemoglobinopathies. Acta haematologica 127, 250-255 (2012).

37. Dharmani, P. et al. Developing a new index and its comparison with other CBC-based indices for screening of beta thalassemia trait in a tertiary care hospital. International Journal of Laboratory Hematology 35, 118 (2013).

38. Pornprasert, S., Panya, A., Punyamung, M., Yanola, J. \& Kongpan, C. Red cell indices and formulas used in differentiation of $\beta$-thalassemia trait from iron deficiency in Thai school children. Hemoglobin 38, 258-261 (2014).

39. Sirachainan, N. et al. New mathematical formula for differentiating thalassemia trait and iron deficiency anemia in thalassemia prevalent area: a study in healthy school-age children. Southeast Asian Journal of Tropical Medicine and Public Health 45, 174 (2014).

40. Bordbar, E., Taghipour, M. \& Zucconi, B. E. Reliability of different RBC indices and formulas in discriminating between $\beta$-thalassemia minor and other microcytic hypochromic cases. Mediterranean journal of hematology and infectious diseases 7 (2015).

41. Janel, A. et al. Proposal of a score combining red blood cell indices for early differentiation of beta-thalassemia minor from iron deficiency anemia. Hematology 16, 123-127 (2011).

42. Keykhaei, B., Rahim, f., Zandian, K. M. \& Pedram, M. Comparison of Different Indices For Better Differential Diagnosis of Iron Deficiency Anemia From B Thalassemia Trait. (2007).

43. Ehsani, M. et al. Discrimination of Iron Deficiency Anemia and Beta Thalassemia Minor Based on a New Index. (2007).

44. Ahmadi, A., Khalilabadi, R., Noorozi, M., Cohan, N. \& Ramzi, M. Evaluation of discrimination indices validity for screening of $\beta$-thalassemia trait. Qom University of Medical Sciences Journal 3, Pe31-Pe36, En35 (2009).

45. Rahim, F. \& Keikhaei, B. Better differential diagnosis of iron deficiency anemia from beta-thalassemia trait. Turk J Hematol 26, 138-145 (2009). 
46. Ghafouri, M., Mostaan, S. L., Sharifi, S., Hosseini, G. L. \& Atar, C. Z. Comparison of cell counter indices in differentiation of beta thalassemia minor from iron deficiency anemia. (2006).

47. Ntaios, G. et al. Discrimination indices as screening tests for $\beta$-thalassemic trait. Annals of hematology 86, 487-491 (2007).

48. Beyan, C., Kaptan, K. \& Ifran, A. Predictive value of discrimination indices in differential diagnosis of iron deficiency anemia and beta-thalassemia trait. European journal of haematology 78, 524-526 (2007).

49. AlFadhli, S. M., Al-Awadhi, A. M. \& AlKhaldi, D. A. Validity assessment of nine discriminant functions used for the differentiation between iron deficiency anemia and thalassemia minor. Journal of tropical pediatrics 53, $93-97$ (2006).

50. Batebi, A., Pourreza, A. \& Esmailian, R. Discrimination of beta-thalassemia minor and iron deficiency anemia by screening test for red blood cell indices. Turkish Journal of Medical Sciences 42, 275-280 (2012).

51. Roth, I. L. et al. Detection of $\beta$-thalassemia carriers by red cell parameters obtained from automatic counters using mathematical formulas. Mediterranean journal of hematology and infectious diseases 10 (2018).

52. Urrechaga, E. \& Hoffmann, J. J. Critical appraisal of discriminant formulas for distinguishing thalassemia from iron deficiency in patients with microcytic anemia. Clinical Chemistry and Laboratory Medicine (CCLM) (2017).

53. Miri-Moghaddam, E. \& Sargolzaie, N. Cut off determination of discrimination indices in differential diagnosis between iron deficiency anemia and $\beta$-thalassemia minor. International journal of hematology-oncology and stem cell research 8, 27 (2014).

54. Matos, J. F. et al. Comparison of discriminative indices for iron deficiency anemia and $\beta$ thalassemia trait in a Brazilian population. Hematology 18, 169-174 (2013).

55. Shen, C. et al. Evaluation of indices in differentiation between iron deficiency anemia and $\beta$-thalassemia trait for Chinese children. Journal of Pediatric Hematology/Oncology 32, e218-e222 (2010).

56. Vehapoglu, A. et al. Hematological indices for differential diagnosis of beta thalassemia trait and iron deficiency anemia. Anemia 2014 (2014).

57. Šimundić, A.-M. Measures of diagnostic accuracy: basic definitions. Med Biol Sci 22, 61-65 (2008).

58. Maroco, J. et al. Data mining methods in the prediction of Dementia: A real-data comparison of the accuracy, sensitivity and specificity of linear discriminant analysis, logistic regression, neural networks, support vector machines, classification trees and random forests. BMC research notes 4,299 (2011).

59. Kim, H. \& Loh, W.-Y. Classification trees with unbiased multiway splits. Journal of the American Statistical Association 96, 589-604 (2001).

60. Kim, H. \& Loh, W.-Y. Classification trees with bivariate linear discriminant node models. Journal of Computational and Graphical Statistics 12, 512-530 (2003).

61. Sharma, S. Applied multivariate techniques. (John Wiley \& Sons, Inc., 1995).

62. Beutler, E. \& Waalen, J. The definition of anemia: what is the lower limit of normal of the blood hemoglobin concentration? Blood 107, 1747-1750, https://doi.org/10.1182/blood-2005-07-3046 (2006).

63. Madan, N., Sikka, M., Sharma, S., Rusia, U. \& Kela, K. Red cell indices and discriminant functions in the detection of betathalassaemia trait in a population with high prevalence of iron deficiency anaemia. Indian journal of pathology \& microbiology 42, 55-61 (1999).

64. Matos, J. F. et al. A new index to discriminate between iron deficiency anemia and thalassemia trait. Revista brasileira de hematologia e hemoterapia 38, 214-219 (2016).

65. Urrechaga, E., Aguirre, U. \& Izquierdo, S. Multivariable discriminant analysis for the differential diagnosis of microcytic anemia. Anemia 2013 (2013).

66. Tripathi, N., Soni, J. P., Sharma, P. K. \& Verma, M. Role of Haemogram Parameters and RBC Indices in Screening and Diagnosis of Beta-Thalassemia Trait in Microcytic, Hypochromic Indian Children. International Journal of Hematological Disorders 2, 43-46 (2015).

67. Piplani, S. et al. Evaluation of various discrimination indices in differentiating Iron deficiency anemia and Beta Thalassemia trait: A practical low cost solution. Annals of Pathology and Laboratory Medicine 3, A551-559 (2016).

68. Ferrara, M., Capozzi, L., Russo, R., Bertocco, F. \& Ferrara, D. Reliability of red blood cell indices and formulas to discriminate between $\beta$ thalassemia trait and iron deficiency in children. Hematology 15, 112-115 (2010).

69. Jameel, T., Baig, M., Ahmed, I., Hussain, M. B. \& Bin Doghaim Alkhamaly, M. Differentiation of beta thalassemia trait from iron deficiency anemia by hematological indices. Pakistan journal of medical sciences 33, 665 (2017).

\section{Acknowledgements}

This work was financially supported by grant no. U-98111 from vice chancellor for Research Affairs of Ahvaz Jundishapur University of Medical Sciences.

\section{Author contributions}

F.R. and M.J. wrote the main manuscript text, and A.S.M. prepared figures and tables and revised the manuscript. All authors reviewed the manuscript.

\section{Competing interests}

The authors declare no competing interests.

\section{Additional information}

Correspondence and requests for materials should be addressed to F.R.

Reprints and permissions information is available at www.nature.com/reprints.

Publisher's note Springer Nature remains neutral with regard to jurisdictional claims in published maps and institutional affiliations.

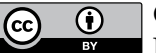

Open Access This article is licensed under a Creative Commons Attribution 4.0 International

License, which permits use, sharing, adaptation, distribution and reproduction in any medium or format, as long as you give appropriate credit to the original author(s) and the source, provide a link to the Creative Commons license, and indicate if changes were made. The images or other third party material in this article are included in the article's Creative Commons license, unless indicated otherwise in a credit line to the material. If material is not included in the article's Creative Commons license and your intended use is not permitted by statutory regulation or exceeds the permitted use, you will need to obtain permission directly from the copyright holder. To view a copy of this license, visit http://creativecommons.org/licenses/by/4.0/.

(c) The Author(s) 2019 